\title{
1 Conserved exchange of paralog proteins during neuronal
}

Domenico Di Fraia ${ }^{1}$, Mihaela Anitei ${ }^{1}$, Marie-Therese Mackmull ${ }^{* 2}$, Luca Parca*3, Laura 5 Behrendt', Amparo Andres-Pons ${ }^{4}$, Darren Gilmour ${ }^{5}$, Manuela Helmer Citterich ${ }^{3}$, Christoph 6 Kaether $^{1}$, Martin Beck ${ }^{6}$ and Alessandro Ori ${ }^{1+1}$

\section{Affiliations}

1 - Leibniz Institute on Aging - Fritz Lipmann Institute (FLI) Beutenbergstraße 1107745 Jena, Germany

2 - ETH Zurich Institute of Molecular Systems Biology Otto-Stern-Weg 3, 8093 Zürich, Switzerland

3 - Department of Biology, University of Tor Vergata, Rome, Italy

4 - European Molecular Biology Laboratory - EMBL, Meyerhofstraße 1, 69117, Heidelberg, Germany

5 - University of Zurich, Department of Molecular Life Sciences, Rämistrasse $71 \mathrm{CH}-8006$ Zürich, Switzerland

6 - Max Planck Institute of Biophysics, department of Molecular Sociology, Max-von-Laue-Straße 3, 60438 Frankfurt am Main

* contributed equally

\# correspondence to alessandro.ori@leibniz-fli.de

\begin{abstract}
Gene duplication enables the emergence of new functions by lowering the general evolutionary pressure. Previous studies have highlighted the role of specific paralog genes during cell differentiation, e.g., in chromatin remodeling complexes. It remains unexplored whether similar mechanisms extend to other biological functions and whether the regulation of paralog genes is conserved across species. Here, we analyze the expression of paralogs across human tissues, during development and neuronal differentiation in fish, rodents and humans. While $\sim 80 \%$ of paralog genes are co-regulated, a subset of paralogs shows divergent expression profiles, contributing to variability of protein complexes. We identify 78 substitutions of paralog pairs that occur during neuronal differentiation and are conserved across species. Among these, we highlight a substitution between the paralogs SEC23A and SEC23B subunits of the COPII complex. Altering the ratio between these two proteins via RNAi-mediated knockdown is sufficient to influence neuron differentiation. We propose that remodeling of the vesicular transport system via paralog substitutions is an evolutionary conserved mechanism enabling neuronal differentiation.
\end{abstract}

Keywords: neuronal differentiation, paralog, development, protein complex, transport, proteome 


\section{Introduction}

A major evolutionary event underlying the emergence of multicellular organisms is the specialization of functions between different cell types. An important role in defining the mechanisms that have led to this diversification is placed on the emergence of specific and definite gene expression programs that characterize distinct cell types (Arendt et al. 2016; Brunet and King 2017). Multicellular organisms are characterized by an increased genome complexity, in part driven by gene duplication events (Ohno 2013; Kaessmann 2010). Indeed paralog genes, namely genes that are the product of gene duplication events, are particularly enriched in the genomes of multicellular organisms (Lynch and Conery 2003). Even though in multicellular organisms the total paralog pool is generally larger, specific cell types express only a limited subset of paralogs, indicating the existence of mechanisms that restrict the expression of some paralogs genes in a given cell type (Padawer, Leighty, and Wang 2012). Most paralog genes share high sequence similarities and regulation of expression (Ibn-Salem, Muro, and Andrade-Navarro 2017). However, cases of divergent expression and regulation have been reported (Soria, McGary, and Rokas 2014;Makova 2003; Assis and Bachtrog 2015; Brohard-Julien et al. 2021), as exemplified by the distinct roles of Hox gene family members in modulating metazoan fronto-caudal development (Ferrier and Holland 2001). More recently, human specific gene duplications have been described to play a role in human brain development (Schmidt et al. 2019; Suzuki et al. 2018). Besides their modulation across cell types, an important role of paralogs is reflected by their ability to compensate for each other in maintaining the general homeostatic state of cells. Genome-wide CRISPR/Cas9-based screens have shown that paralog genes have a protective action on cell proliferation against the effect of gene loss-of-function in humans (Dandage and Landry 2019) and cancer cell lines (De Kegel and Ryan 2019; Thompson et al. 2021). All these observations highlight the functional impact that paralog genes have in modulating biological activity, development and cell differentiation.

From a molecular point of view, paralogs have been shown to modulate biological processes by influencing the assembly and activity of protein complexes. We have previously shown that specific compositions of protein complexes can be identified across cell types (Ori et al. 2016), and individuals (Romanov et al. 2019), and that the exchange of paralog complex members can contribute in specific cases to this variability. It has been also shown that the alternative incorporation of paralog proteins can antagonistically modulate the function of some protein complexes. For example, multiple specific paralog substitutions between subunits of the BAF chromatin remodelling complex lead to the assembly of functionally distinct complexes that can influence pluripotency and neuronal differentiation (Son and Crabtree 2014; Ho et al. 2009; Kaeser et al. 2008). Similarly, ribosomal paralog proteins promote ribosome modularity (Shi et al. 2017) and directly affect mRNA translation specificity (Gerst 2018; Slavov et al. 2015; Genuth and Barna 2018). Finally, co-expression analysis of protein complex members during human keratinocyte differentiation highlighted the existence of paralog subunits that compete for the same binding site in variable complexes (Toufighi et al. 2015). These studies indicate that paralog genes can contribute to the instalment of specific biological functions required, e.g., for cell differentiation, by influencing the activity of specific protein complexes. It remains currently unclear whether similar mechanisms extend to other molecular networks across the proteome and to which extent the regulation of paralog expression is conserved across cell types of different species.

In this study, using both newly generated and publicly available datasets, we systematically investigate how the expression of paralog genes contributes to transcriptome and proteome 
diversification across tissues, during development and neuronal differentiation. By integrating data from multiple organisms, we define a specific signature of paralog genes that emerges during neuronal differentiation and is conserved from fish to human.

\section{Results}

\section{Co-expression of paralog genes during embryo development and across human tissues}

In order to study the contribution of gene duplication to cell and tissue variability, we analyzed the expression profiles of paralog genes during zebrafish embryonic development and across healthy human tissues. We took advantage of two publicly available datasets describing a time-course transcriptome of zebrafish embryo development (White et al. 2017), and the steady state transcriptomes and proteomes of 29 healthy human tissues (Wang et al. 2019). We used correlation analysis of transcripts and proteins encoded by paralog genes to address their co-regulation during development and in fully differentiated tissues. According to Ensembl Compara (Yates et al. 2020) roughly $70 \%$ of the protein coding genes in the zebrafish and human genomes have paralogs, and similar proportions of paralogs are reflected in the datasets considered in this study $(71 \%$ and $74 \%$ for zebrafish and human, respectively) (Fig1A). During zebrafish embryo development and across human tissues the majority of paralog genes pairs tend to be positively correlated $(R>0)($ Table S1), however, a substantial proportion of them ( $33 \%$ and $36 \%$ for development and tissue, respectively) appears to be coregulated in a negative manner $(R<=0)(F i g 1 B, C)$. Comparable results were found also at the protein level across tissues, where the proportion of differently regulated paralog proteins appeared to be even higher (48\%) (Supp.Fig1A). By calculating coefficient of variations for each protein and transcript, we also noticed that genes that possess paralogs in the genome tend to be more variably expressed during development (Supp.Fig1B) (two-sided Wilcoxon test $p<2.2 \mathrm{E}-16$ ), and across differentiated tissues at both transcriptome (Supp.Fig1C) (twosided Wilcoxon test $\mathrm{p}<2.2 \mathrm{E}-16$ ) and proteome level (Supp.Fig1D) (two-sided Wilcoxon test $\mathrm{p}<2.2 \mathrm{E}-16)$.

Since substitution of paralog members can contribute to the functional specialization of large protein complexes, such as chromatin remodeling complexes and ribosomes (Ori et al. 2016; Toufighi et al. 2015; Slavov et al. 2015; Romanov et al. 2019), we focused on the analysis of paralog expression in the context of protein complexes. We observed a characteristic behaviour of paralog pairs that assemble in the same protein complex. While paralogs coexpression was generally positively related to their sequence identity, i.e., highly similar paralogs tended to be co-regulated $(R=0.16$, Pearson correlation $p=<2.2 \mathrm{E}-16$ for development; $R=0.33$ Pearson correlation $p<2.2 E-16$ for tissues), this was not the case for paralog pairs residing in the same protein complex, $(R=-0.11$, Pearson correlation $p=3.69 E-$ 05 for development; $R=-0.03$, Pearson correlation $p=0.19$ for tissues, Fig1D, Fig1E, Table S1). This underlines the existence of a subset of paralog pairs that display no or negative coexpression, despite being members of the same protein complex and sharing a high sequence identity.

In order to estimate the contribution of these paralog genes to context-dependent protein complex formation, we investigated variations in the composition of macromolecular complexes during development and across tissues. We calculated the median correlation between all the possible pairs of genes belonging to the same protein complex and selected 
142 the upper and lower $25 \%$ percentiles of the resulting distribution to classify protein complexes

143 as stable or variable, respectively (Supp.Fig2A, Table S2). During zebrafish development, we

144 observed, as expected, positive correlations between protein complex members (Supp. Fig2B,

$145 \mathrm{p}<2.2 \mathrm{E}-16$, two-sided Wilcoxon test). Highly correlated complexes include large house-

146 keeping complexes, e.g., ribosomes and the proteasome, while functions carried out by more

147 variable ones included molecular motors like the dynein-complex, vesicle associated proteins,

148 e.g., SNARE, COPII/coat protein complex II, and chromosome and chromatin regulators, e.g.,

149 chromatin structure remodeling (RSC) complex (Supp.Fig2B, Table S2). The contribution of

150 paralogs genes to the observed variability of protein complexes is highlighted by a general

151 positive correlation between protein complex variability and paralog content, i.e., the fraction

152 of complex members that have at least one paralog in the genome $(R=0.40, p=2.1 E-10)$

153 (Fig1F, Table S2). A similar pattern can be observed across human tissues at both

154 transcriptome $(R=0.23, p=7.9 E-05$, Fig1G, Table $S 2)$ proteome $(R=0.27, p=1.3 e-05)$ levels

155 (Supp.Fig2C, Table S2). By calculating co-expression of single subunits (Supp.Fig2D), we

156 consistently observed that complex members that possess at least one paralog tend to have

157 a more variable expression compared to other members of the same complex (Supp.Fig2E,

158 F, G, Table S2).

159

160 Interestingly, some of the most enriched Gene Ontology terms (GO) among anti-correlated 161 paralog subunit pairs (bottom $25 \%$ of the distribution) were related to vesicle mediated 162 transport and protein localization (Fig1 $\mathrm{H}$, Table S3), suggesting a potential divergent role of 163 paralog proteins in establishing or modulating these biological functions. Our analysis 164 recapitulated known anti-correlated expression for paralogs that are part of the BAF chromatin 165 remodelling complex (homologous of the yeast SWI/SNF complex (Xue et al. 2000)) (Hansson 166 et al. 2012; Ori et al. 2016; Ho et al. 2009) (Fig1l), but also specific expression profiles for members of the histone acetyl-transferase complex HBO1 (Fig1J), among others (Table S1).

168 Similar expression patterns were observed also for paralogs belonging to complexes involved in the intracellular transport of macromolecules, such as the COPI and COPII complexes (Fig1K, Fig1L). Together these data suggest the existence of an evolutionary pressure for paralog subunits, especially involved in molecular trafficking and chromatin remodelling, to conserve sequence identity while diverging in expression across developmental stages and differentiated tissues. 
A

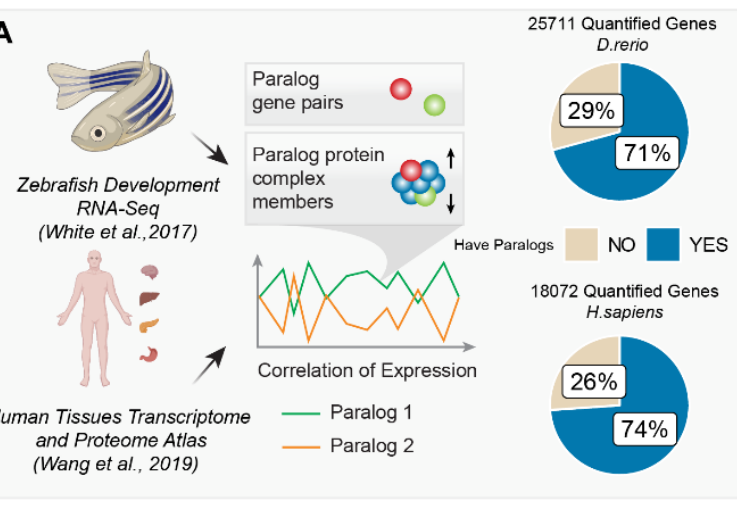

D

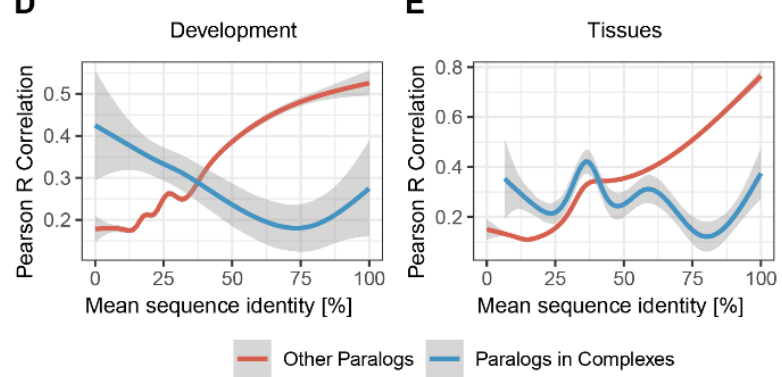

H

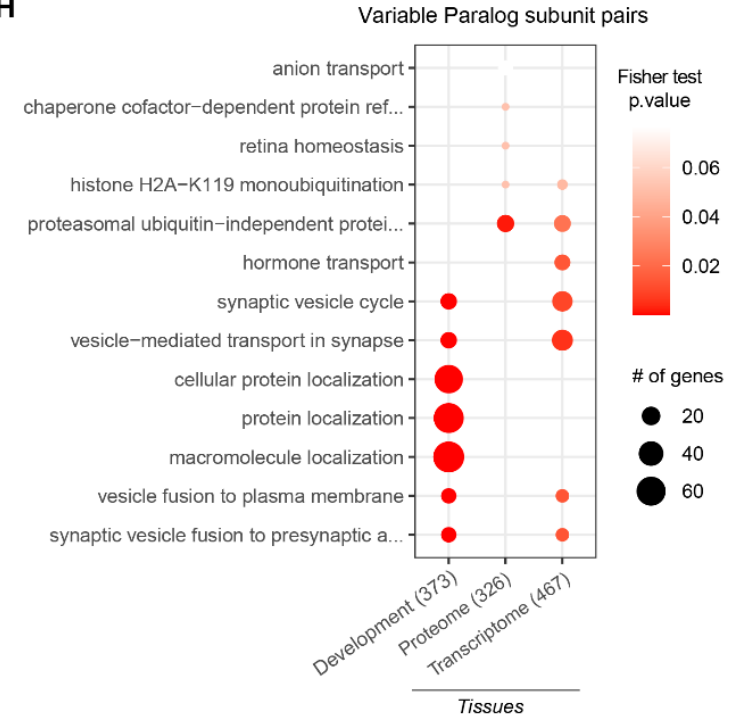

B Correlation of Paralog Pairs - Development

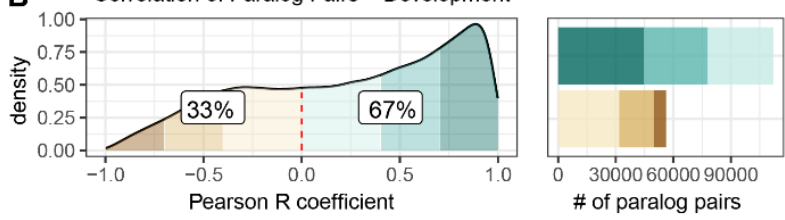

C Correlation of Paralog Pairs - Tissues - Transcriptome

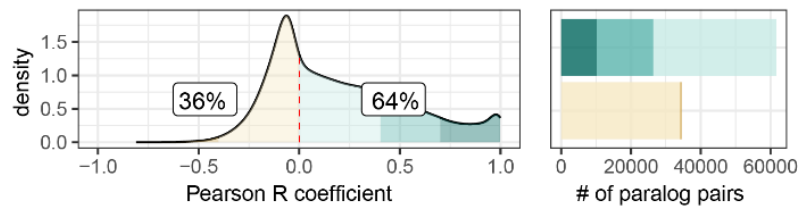

$\mathbf{F}$

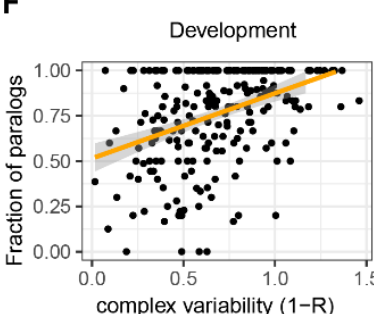

G

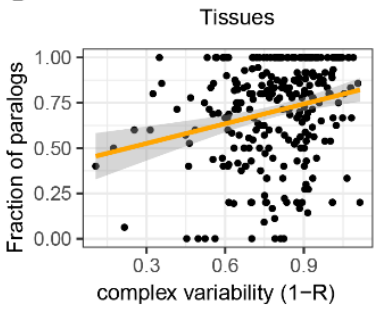

I
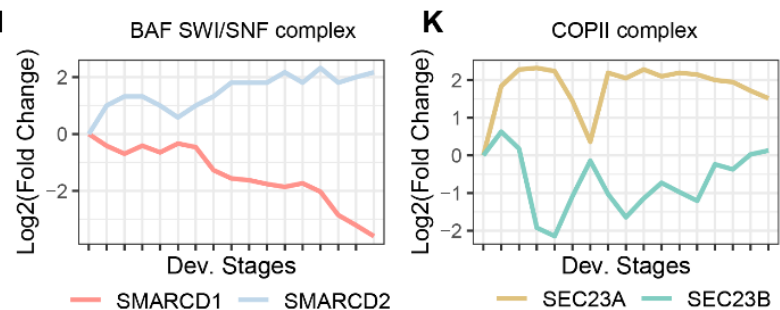

- SMARCD1 - SMARCD2

- SEC23A - SEC23B
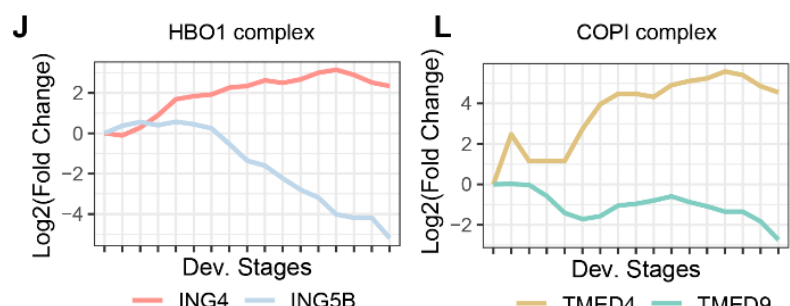

A - Transcriptome data during zebrafish embryo development (White et al. 2017) and transcriptome and proteome data from 29 healthy human tissues (Wang et al. 2019) were used to calculate Pearson correlation of expression during development and across tissues for paralog gene pairs. Pie plots indicate the proportion of quantified transcripts that possess at least one paralog in the zebrafish and human dataset, respectively.

B/C - Density distribution of paralog gene pairs Pearson correlations during zebrafish embryo development (B) and across human tissues (C). Colored areas highlight different correlation intervals. Labels indicate the percentage of paralogs that are positively correlated $(R>0)$ and negatively correlated $(R<=0)$. Barplot indicates the number of paralog pairs present in each category. Transcriptome data were used for both comparisons.

D/E - Generalized Additive Model representing the relationship between mean paralog pairs reciprocal identity and transcript Pearson correlation in zebrafish development $(D)$ and human tissues $(E)$. Colored lines indicate paralog pairs that are members of the same protein complex (blue), and all other paralog pairs (red). 
bioRxiv preprint doi: https://doi org/101101/2021.07.22 453347; this version posted July 23,2021 . The copyright holder for this preprin (which was not certified by peer review) is the author/funder, who has granted bioRxiv a license to display the preprint in perpetuity. It is made available under aCC-BY-NC-ND 4.0 International license.

190 F/G - Relationship between paralogs content (fraction of subunits that have paralogs in the genome) 191 and complex variability. Complex variability is expressed as $1-R$, where $R$ is the median Pearson 192 correlation of expression between all complex subunits. Transcriptome data were used for both embryo 193 development $(F)$ and tissues $(G)$.

$194 \mathrm{H}-\mathrm{GO}$ term over representation analysis for the $25 \%$ most variable paralog subunit pairs against all 195 other paralog subunit pairs. The top 5 most enriched terms from each dataset (development, tissues's 196 transcriptome, and tissues's proteome) are shown. Numbers in parentheses on the x-axis indicate the 197 number of unique variable paralog pairs considered for enrichment.

198 I-L - Transcriptome profiles along embryo development for specific paralog pairs part of chromatin 199 organization complexes, BAF SWI/SNF (I) and HBO1 (J), or vesicle-transport complexes, COPII (K) 200 and COPI (L). Log2 fold changes calculated from TPMs relatively to the first time point are shown.

A

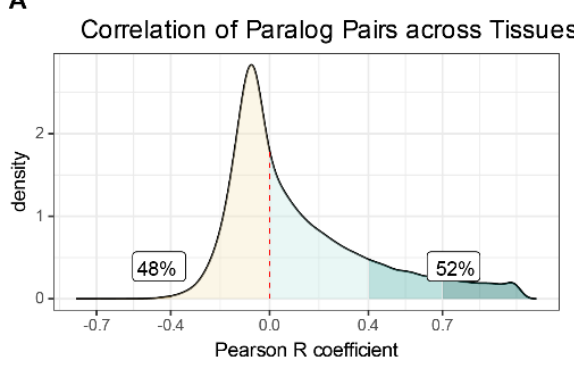

C Tissues - Transcriptome Wilcox pvalue $4.55 \mathrm{e}-58$

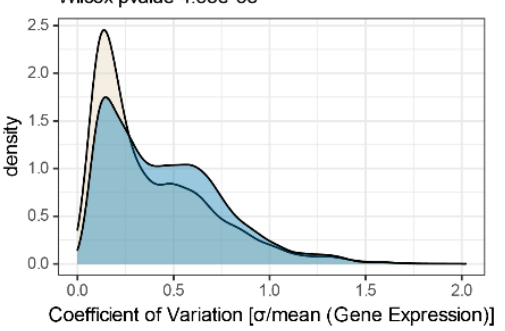

Coefficient of Variation [ $\sigma /$ mean (Gene Expression)]
B Development

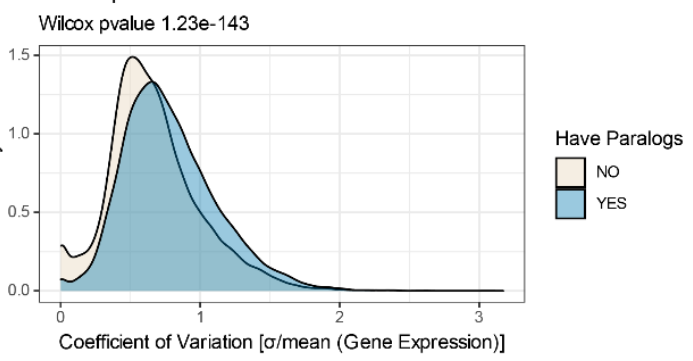

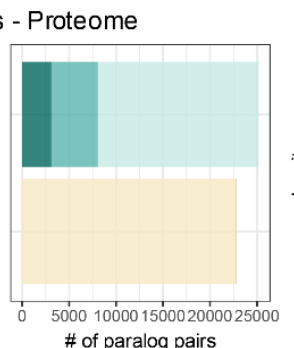

D Tissues - Proteome

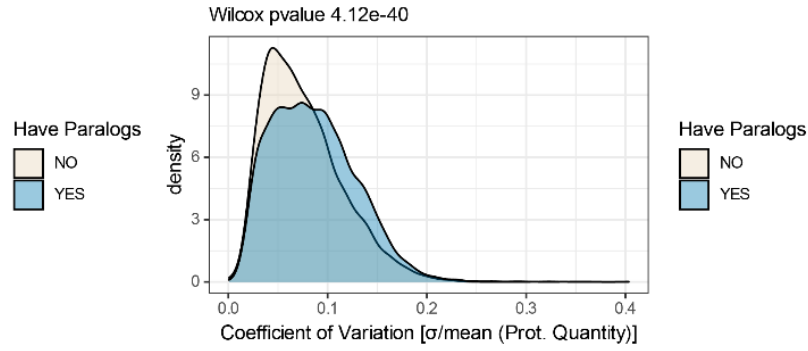

\section{Supp. Fig1 - Paralogs contribute to transcriptome and proteome variability during development} and across tissues

A - Density distribution of paralog protein pairs Pearson correlation across human tissues. Colored areas highlight specific correlation intervals. Labels indicate the percentage of paralogs that are positively correlated $(R>0)$ and negatively correlated $(R<=0)$. Barplots indicate the numbers of paralog pairs present in each category.

B-D - Coefficient of variation of genes that have paralogs (blue) and genes that do not have any (grey). Density distributions are shown for Zebrafish development (B), transcript across tissues (C) and protein across tissues (D). 


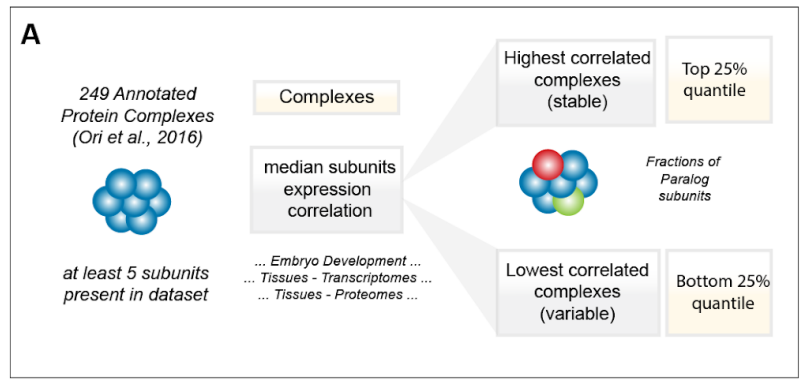

B Complexes Random Complexes

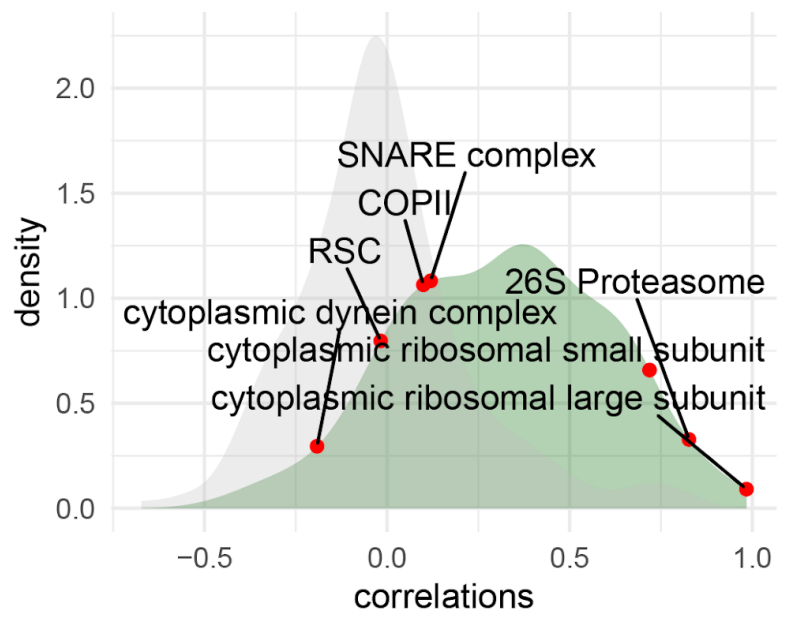

\section{c Tissues - Proteome}

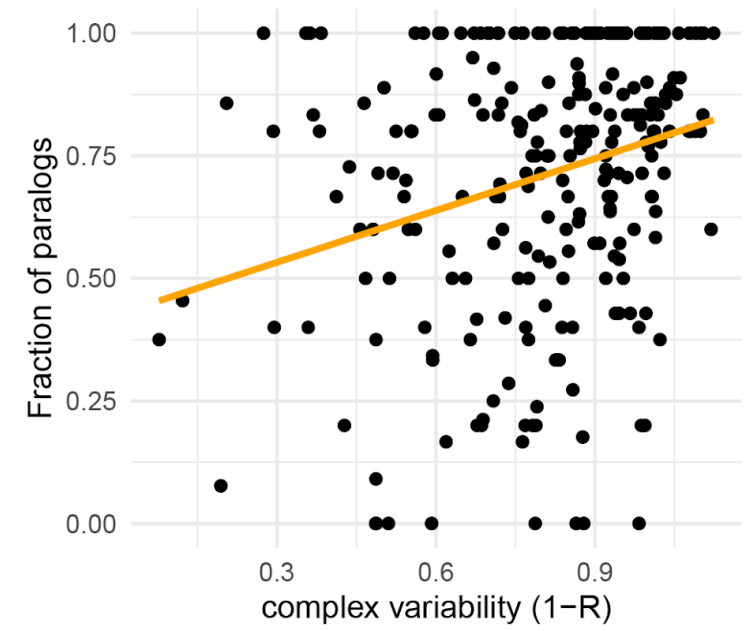

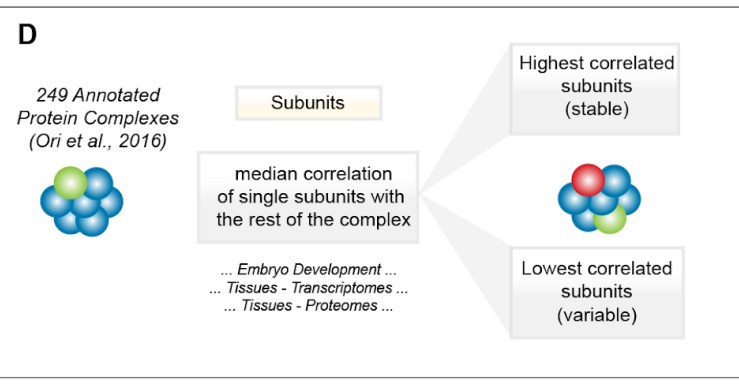

$E$ Development

Wilcox pvalue $=1.4 \mathrm{e}-31$

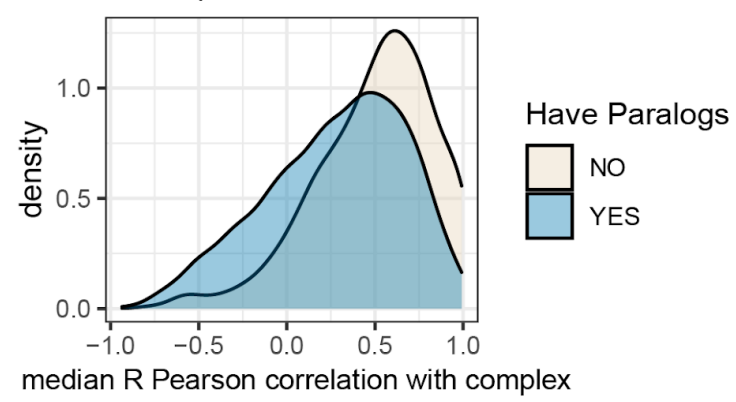

Tissues Transcriptome

F

Wilcox pvalue $=3.7 \mathrm{e}-14$

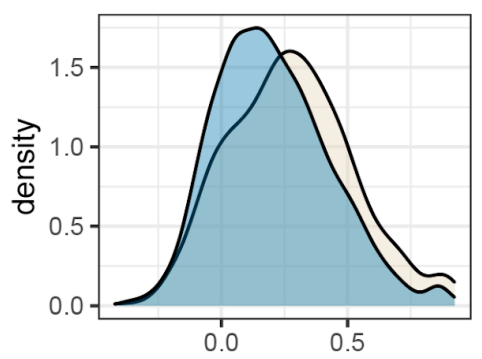

median R Pearson correlation with complex

Tissues Proteome

G

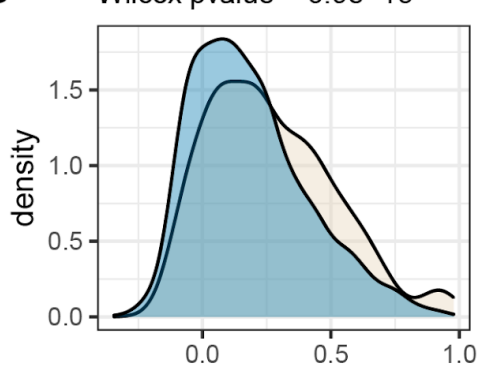

median R Pearson correlation with complex

\section{Supp. Fig2 - Paralogs contribute to the variability of protein complexes}

215 A - Scheme of the protein complex analysis workflow.

B - Density distributions of median Pearson correlation coefficient of the zebrafish development data for protein complexes (green) and a randomized set of protein complexes (grey) obtained by randomly assigning genes to protein complexes of the same size as the original set. Selected complexes displaying high or low correlations are highlighted on the distribution.

$C$ - Relationship between paralogs content (fraction of subunits that have paralogs in the genome) and complex variability. Complex variability is expressed as $1-R$, where $R$ is the median Pearson correlation of expression between all complex subunits. Based on proteome data from human tissues. 
D - Scheme of the Analysis - For each subunit of the 249 protein complexes in each dataset, we calculated the median Pearson correlation coefficient with all the other subunits of the same complex. The median Pearson correlation coefficient is used to assess whether each subunit is stoichiometrically stable (high correlations) or variable (low correlations).

E-G - Distributions of median Pearson correlation coefficient for single subunits against their complex. Subunits with paralogs (blue) show lower median correlation with their complex compared to subunits that do not have paralogs (grey). Results are shown for zebrafish embryo development (E), transcriptome $(\mathrm{F})$ and proteome $(\mathrm{G})$ across tissues.

231

232

233

234

235

236

237

238

239

240

241

242

243

244

245

246

247

248

249

250

251

252

253

254

255

256

257

258

259

260

261

262

263

264

265

266

267

268

269

270

271

\section{Conserved exchange of paralog proteins during neuronal differentiation.}

To investigate in more detail how the alternative usage of paralog genes contributes to cell variability, we focused on the well characterized process of neurogenesis that has been studied across different species by genome-wide approaches using both in vivo and in vitro model systems. We analysed neurogenesis datasets from zebrafish, mouse, rat and human (Fig2A, Table S4), based on the hypothesis that if some particular paralog substitutions are conserved across multiple organisms, they are more likely to functionally contribute to this process. We decided to use proteomics data to account for both transcriptional and post transcriptional mechanisms regulating paralog abundances.

We generated a proteomic dataset using mouse primary neurons harvested after 0,3 and 10 days of in vitro differentiation (DIV0, DIV3, DIV10). Shortly, cortical immature neurons were isolated from wild-type embryonic (E15.5) mouse brains and differentiated in glia-conditioned neurobasal medium. Neurons were collected at different time points and analysed by quantitative mass spectrometry (see Methods for details). We integrated this dataset with comparable data obtained from rat and human (Frese et al. 2017; Djuric et al. 2017). The rat dataset consisted of a time-course analysis of in vitro neurogenesis similar to the one performed in mouse, while the human data compared induced pluripotent stem cells (iPSC), iPSC-derived neural progenitor cells (NPCs) and cortical neurons (Neu). Finally, to directly compare the proteomes of embryonic stem cells and in vivo differentiated neurons, we took advantage of an established zebrafish line that enables the isolation of intact neurons using a fluorescent reporter. In this fish strain, the red-fluorescent-protein dsRed is expressed under the control of a neuronal-specific tubulin promoter from Xenopus (NBT-dsRed) (Peri and Nüsslein-Volhard 2008), allowing the selective isolation of neuronal cells by fluorescenceactivated cell sorting (FACS). Undifferentiated cells were extracted from wild-type zebrafish 6 hours post fertilization (hpf), while NBT-dsRed zebrafish 1 day post fertilization (dpf) were used for the collection of differentiated neurons.

The quality of each dataset was evaluated using Principal Component Analysis (PCA) and GO enrichment analysis, confirming data reproducibility across replicates and the expected enrichment of terms related to neuronal development and cell differentiation (Supp.Fig3A, B, $C$, D). The neuronal differentiation data recapitulated the general patterns of paralog expression that we observed during development and across tissues: (i) proteins that have at least one paralog in the genome displayed larger fold changes (Fig2B); (ii) paralog pairs were generally co-regulated (Supp.Fig4A, Table S5); (iii) a subset of paralog pairs ( 20\%) displayed opposite regulation (Fig2C, Table S5). The latter set of paralogs was enriched for proteins related to chromatin remodeling, RNA splicing, RAS signalling, exocytosis and vesicle transport, as well as other processes related to development. Interestingly, while some enrichments were dataset-specific, we consistently observed an enrichment of GO terms 
272 related to DNA binding and transport across all datasets (Supp.Fig4B, Table S5). The

273 neuronal differentiation datasets confirmed that paralogs contribute to protein complex

274 variability, since in general, proteins that have at least one paralog display higher

275 stoichiometric variability (Fig2D, Table S6), and, consequently, variable complexes were

276 enriched in proteins with at least one paralog.

277

278 We then focused our analysis on paralog pairs that displayed divergent abundance changes 279 during the neuronal differentiation process. In order to capture more subtle changes, we 280 analyzed ratios between pairs of paralogs across conditions using absolute protein amounts 281 estimated from mass spectrometry data. Briefly, log2 abundance ratios were calculated for all 282 possible eggNOG (Huerta-Cepas et al. 2019) pairs across conditions, and significant changes 283 in these ratios were statistically assessed using a linear model (see Methods) (Table S7). 284 Differences in paralog ratios were sufficient to describe the general structure of the data, as 285 highlighted by the separation of human, rodent and zebrafish dataset by PCA (Fig3A). By 286 mapping every paralog pair to its relative eggNOG, we compared differences in paralog ratios 287 across datasets. We were then able to assess which specific changes are conserved across 288 the species considered. By applying a stringent cut-off (Log2 paralog ratio differences 289 consistent in direction in all species and at least in 5 of the 7 conditions considered, and 290 combined adjusted $p<=0.05$, see Methods), we identified 78 paralog eggNOG pairs 291 consistently affected during neuronal differentiation across all the species tested (Fig3B, Table 292 S7). These conserved paralog pairs included multiple proteins involved in redox metabolism, 293 RNA splicing, vesicles mediated trafficking and transport. Specifically, we found changes in 294 ratios between the COPII complex subunits such as SEC23A and SEC23B (Fig3C), 295 components of the retromer complex (VSP26B and VPS26A) (Supp.Fig5A), dynein subunits 296 (DYNC1LI1 and DYNC1LI2) (Supp.Fig5B), and GTPase regulators of vesicle trafficking 297 (RAB14 and RAB8A) (Supp.Fig5C). Taken together, these data highlight a potential role for 298 paralogs proteins in mediating modularity of protein complexes during neuronal differentiation. 299 Highly conserved substitutions between paralogs appear to predominantly affect paralog pairs 300 that participate in the formation of transport complexes. This suggests that these substitutions 301 might be required to adapt the transport system during neuronal differentiation and 302 development in general. 
bioRxiv preprint doi: https://doi.org/10.1101/2021.07.22.453347; this version posted July 23,2021 . The copyright holder for this preprint (which was not certified by peer review) is the author/funder, who has granted bioRxiv a license to display the preprint in perpetuity. It is made available under aCC-BY-NC-ND 4.0 International license.

A
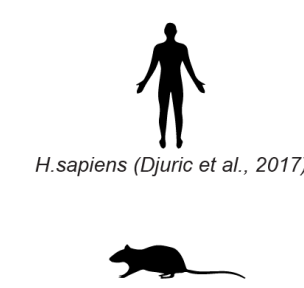

R.norvegicus (Frese et al., 2017)
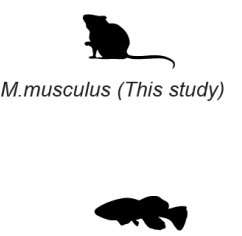

D. rerio (This study)

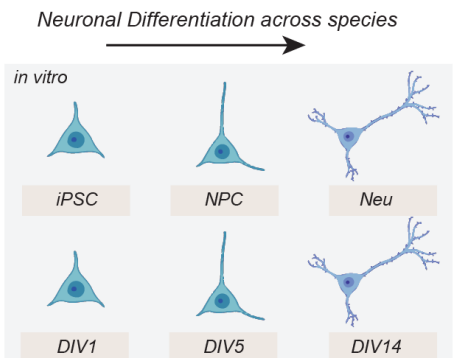

DIV5
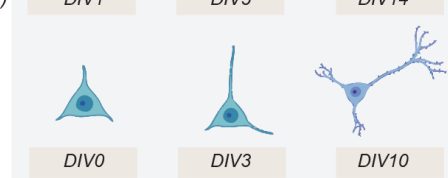

DIV10

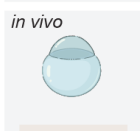

Stem
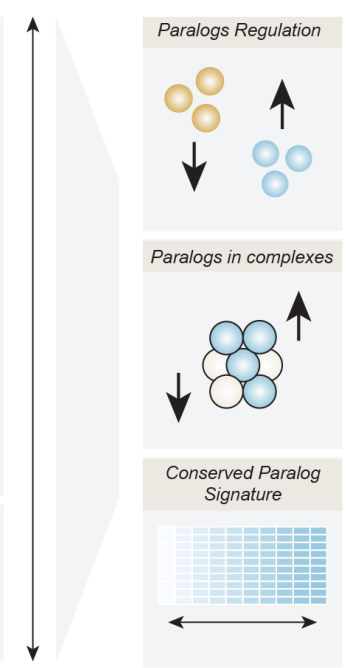

Paralogs in complexes

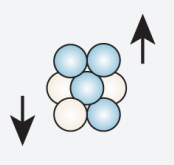

Conserved Paralog Signature

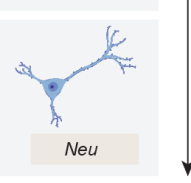

Abundance changes during neurogenesis

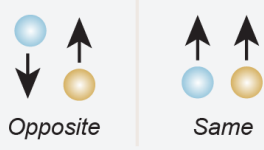

Co-expression of subunits

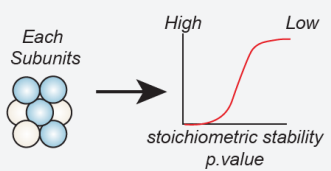

Change of paralogs ratio

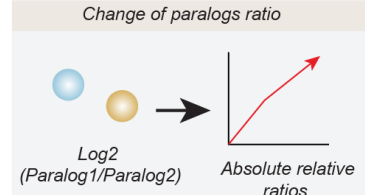

B Have Paralogs 官 NO 官 YES
C

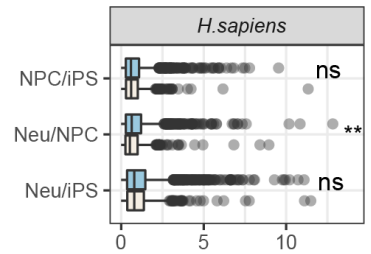

$\begin{array}{llllll}0.0 & 2.5 & 5.0 & 7.5 & 10.0 & 12.5\end{array}$

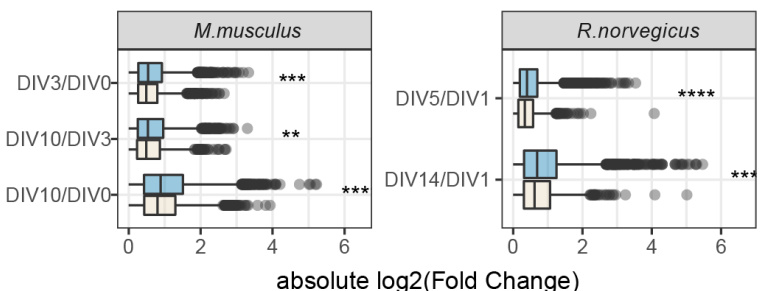

absolute log2(Fold Change)
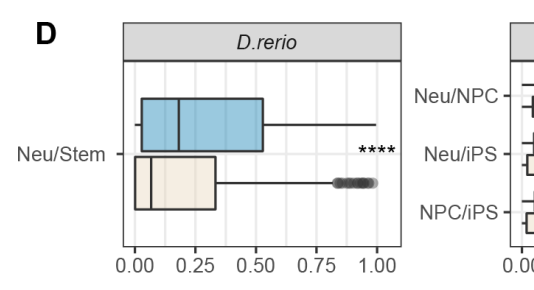

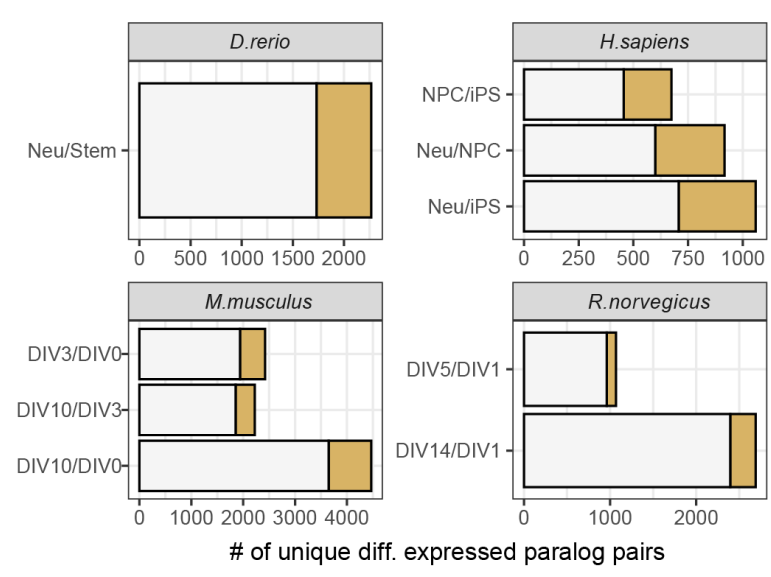

\section{Fig2 - Changes of abundance of paralog proteins during neuronal differentiation}

A - Overview of dataset used and data analysis workflow. DIV=differentiation in vitro day, iPSC=induced pluripotent stem cell, Neu=Neurons, NPC neuronal precursor cell, Stem=undifferentiated stem cell. B - Boxplots display absolute Log2 fold changes during neuronal differentiation for proteins that have (blue) or do not have (grey) at least one paralog.

C - Barplots show the numbers of unique paralog pairs regulated in a concordant (grey) or opposite direction (orange) during neuronal differentiation.

D - Boxplots compare the stability of protein complex subunits that have (blue) or do not have (grey) at least one paralog in the same protein complex. Low $p$ values indicate subunits that are significantly coexpressed with the other members of the same protein complex and are therefore considered as "stable". In B and D, asterisks indicate $p$ values of the two-sided Wilcoxon test between the two compared groups: * $p<=0.05 ;{ }^{* *} p<=0.01,{ }^{* * *} p<=0.001,{ }^{* * * *} p<=0.0001$, ns $=$ not significant. 
bioRxiv preprint doi: https://doi.org/10.1101/2021 07 22.453347; this version posted July 23, 2021. The copyright holder for this preprin (which was not certified by peer review) is the author/funder, who has granted bioRxiv a license to display the preprint in perpetuity. It is made available under aCC-BY-NC-ND 4.0 International license.

A

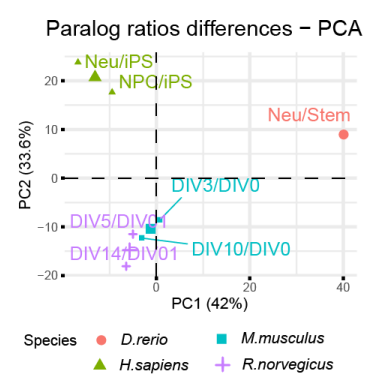

B
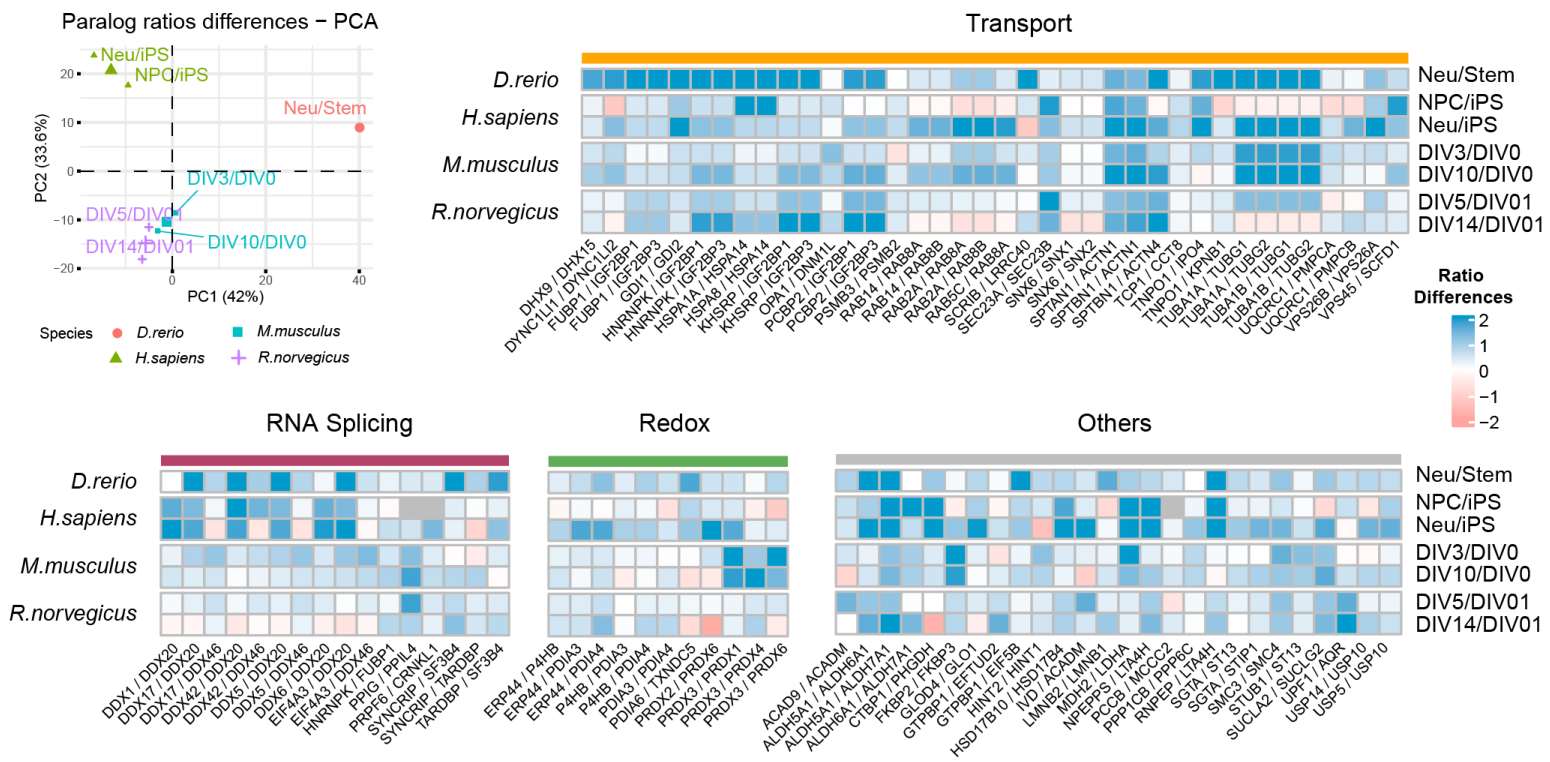

C
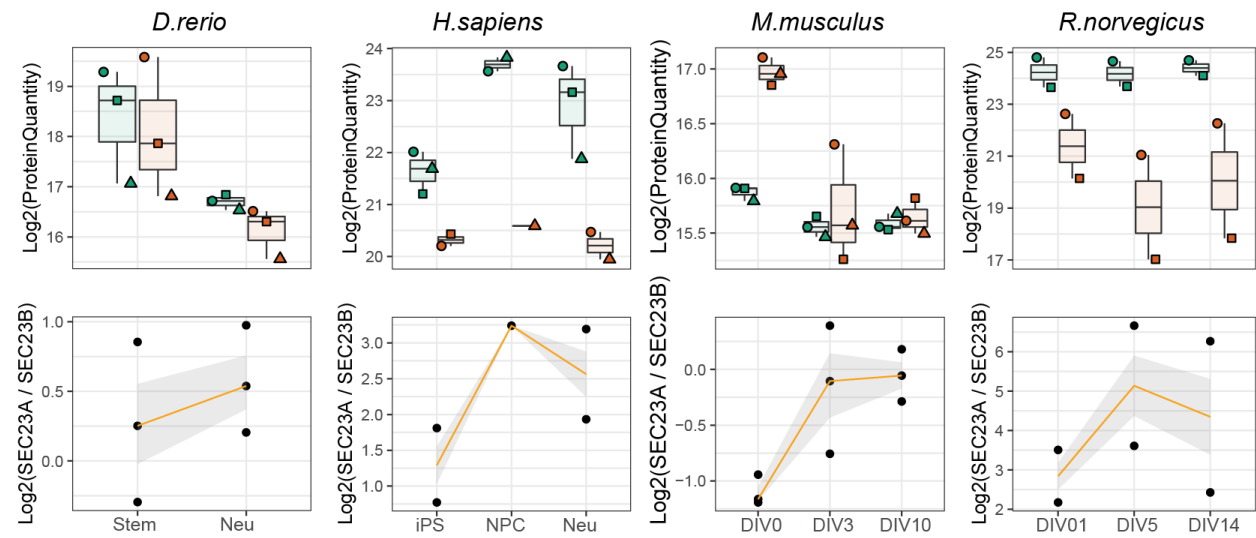

SEC23A

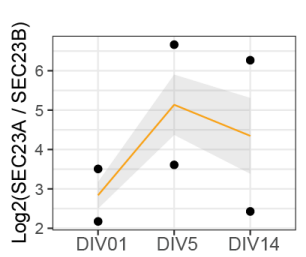

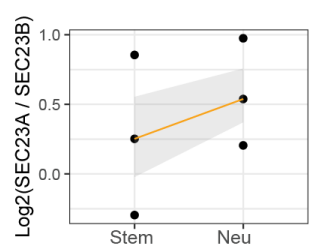

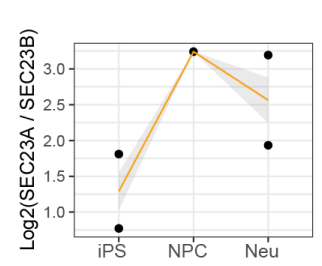

Fig3 - A conserved paralog signature during neuronal differentiation

A - Principal Component Analysis based on paralog ratio differences across conditions. Only paralog ratios quantified in all datasets are used for the analysis. The color code indicates the different species analysed, the small symbols indicate the different comparisons tested, and the large symbols indicate the centroid for each species.

B - Heatmap shows conserved paralog substitutions during neuronal differentiation. Each column represents a specific eggNOG paralog pair mapped to the same human genes. Grey tiles indicate paralog pairs not quantified in the given condition. Paralog pairs are grouped according to their known biological function.

C - Protein abundance profiles for SEC23A (green) and SEC23B (orange) across datasets. Boxplots indicate Log2 protein quantities, across different replicates, while line plots (bottom) indicate the ratios between the two paralogs. In the top panel, shapes indicate paired replicate experiments. In the bottom panel, orange lines indicate the mean paralog ratio across replicates, and the shaded area represents $50 \%$ confidence intervals. 
bioRxiv preprint doi: https://doi.org/101101/2021.07.22.453347; this version posted July 23, 2021. The copyright holder for this preprint (which was not certified by peer review) is the author/funder, who has granted bioRxiv a license to display the preprint in perpetuity. It is made available under aCC-BY-NC-ND 4.0 International license.

A

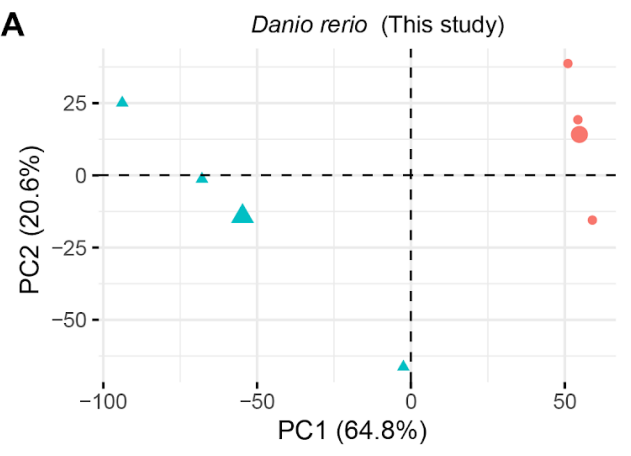

Groups $\bigcirc \mathrm{Neu} \triangle \mathrm{Stem}$
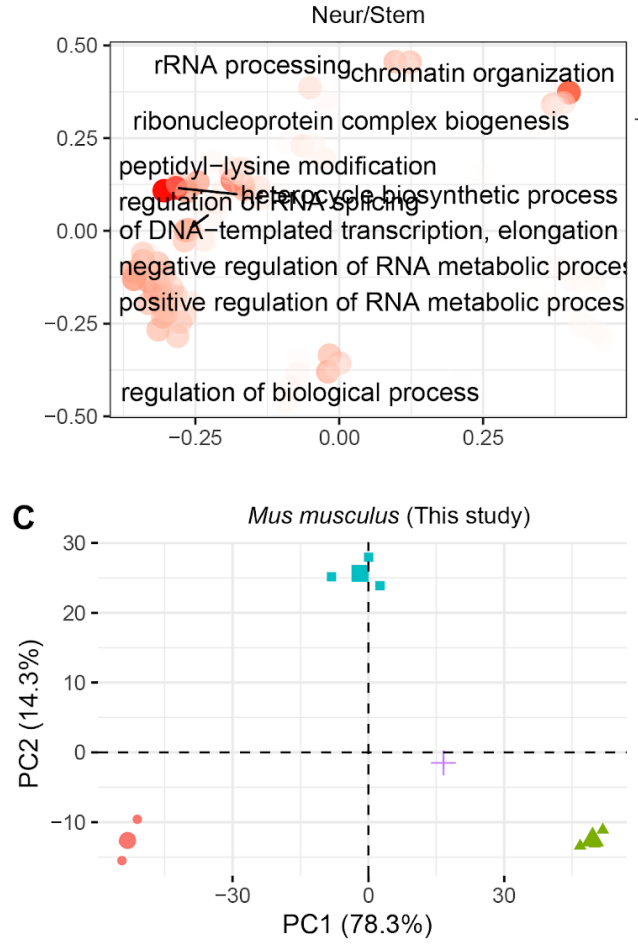

Groups $\square$ DIV0 $\Delta$ DIV10 $\square$ DIV3 + Poo

DIV10/DIV0

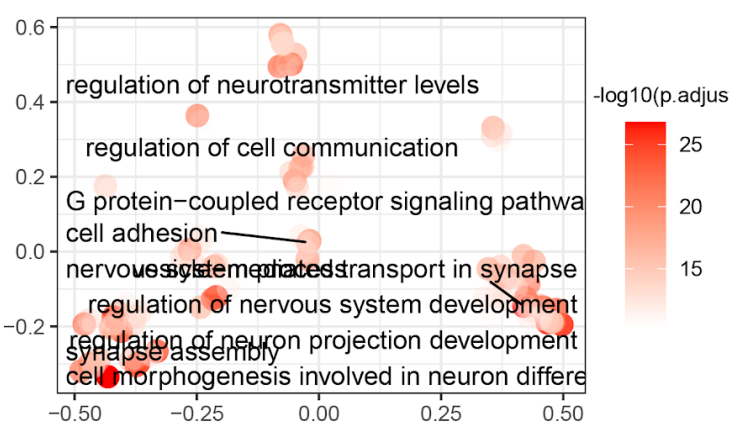

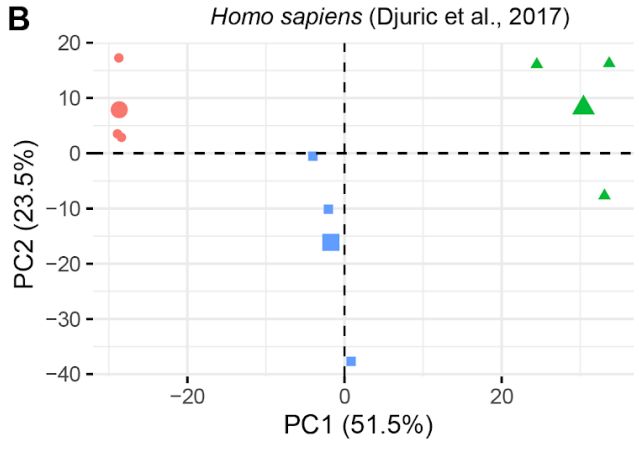

Groups $\bigcirc$ iPs $\Delta$ Neu $\square$ NPC

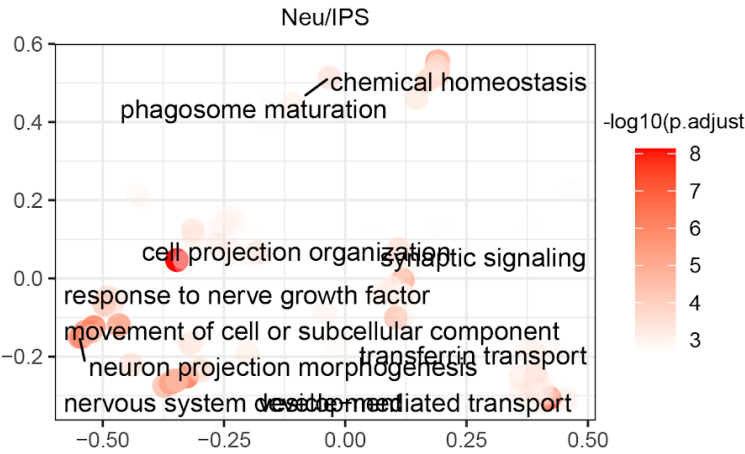

D

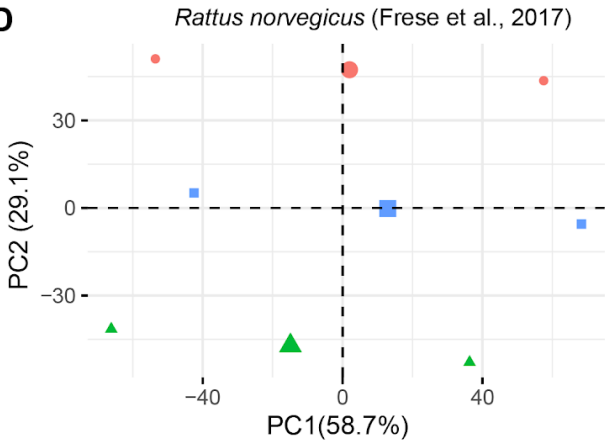

$\begin{array}{llllll}\text { Groups } & \text { DIV1 } & \mathbf{\Delta} & \text { DIV14 } & \mathbf{\square} & \text { DIV5 }\end{array}$

DIV14/DIV1

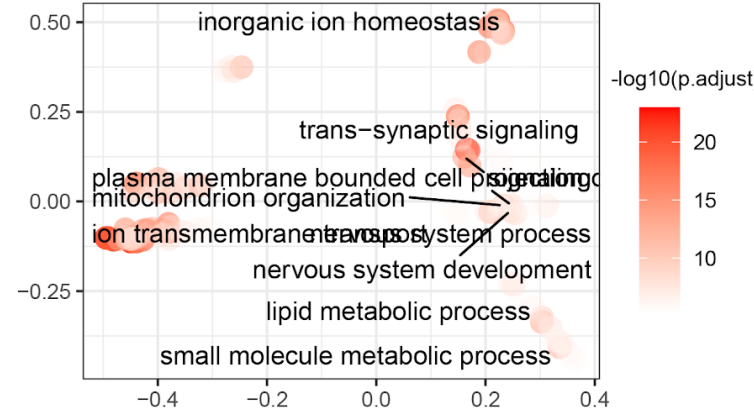

\section{Supp. Fig3 - Proteome data of neuronal differentiation}

A-D - Principal Component Analysis (PCA) of the proteomics data used in this study. Small symbols indicate the different replicates, large symbols indicate the centroid of each condition. Color and symbols indicate the different conditions considered. Below each PCA plot, the over representation analysis for "Biological Process" GO terms enriched among upregulated proteins (Log2 fold change $>=0.58$ ) against the rest of the quantified proteins is shown. In each plot, the $\mathrm{x}$ and $\mathrm{y}$ axis indicate 
bioRxiv preprint doi: https://doi.org/10.1101/2021.07 22.453347: this version posted July 23, 2021. The copyright holder for this preprint (which was not certified by peer review) is the author/funder, who has granted bioRxiv a license to display the preprint in perpetuity. It is made available under aCC-BY-NC-ND 4.0 International license.

semantic space separating the different terms, the color scale indicates the -log10(adjusted $p$ value) of the Fisher test. DIV= differentiation in vitro day, iPSC=induced pluripotent stem cell, Neu= Neurons, $\mathrm{NPC}=$ neuronal precursor cell, Stem $=$ undifferentiated stem cell.

A
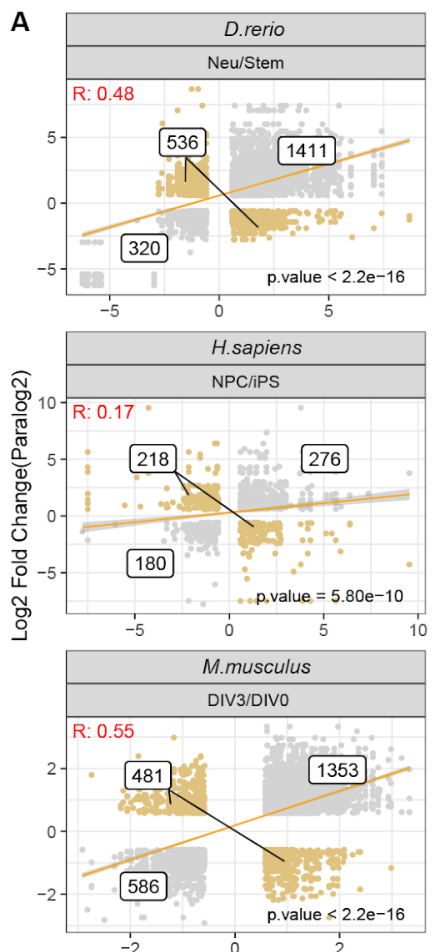
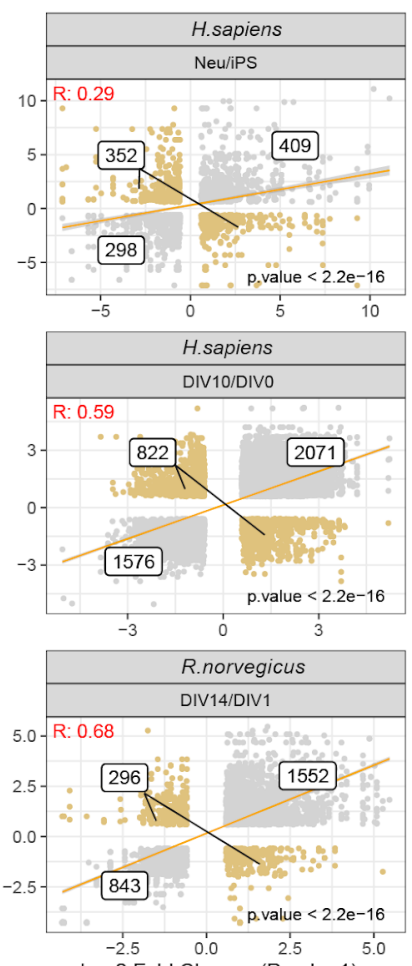
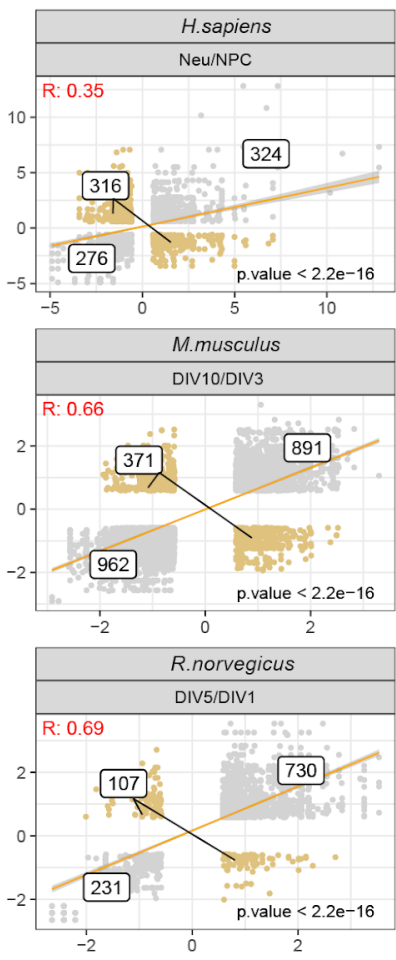

B

Log2 Fold Change (Paralog1)

Transport,synapse, vesicles DNA and chromatin

others

\begin{tabular}{|c|c|c|}
\hline & H.sapiens & \\
\hline NPC/PS & Neu/IPS & $\mathrm{Neu} / \mathrm{NP}$ \\
\hline $\begin{array}{l}25 \% \\
5 \% \quad 70 \%\end{array}$ & $\begin{array}{l}23 \% \\
7 \% \quad{ }_{70 \%}\end{array}$ & $27 \%$ \\
\hline
\end{tabular}
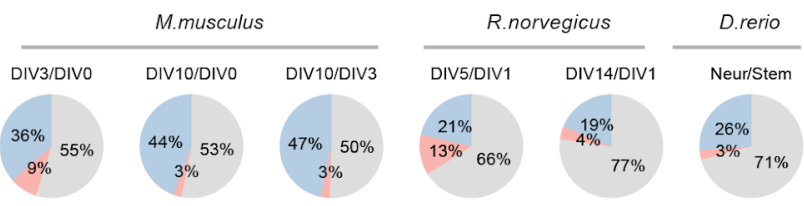

ion transport

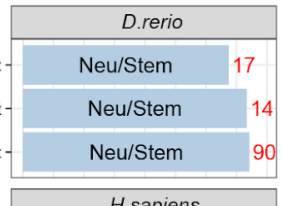

DNA unwinding involved in DNA replicatio

transmembrane transport

transport -

H.sapiens

endosomal transport

vesicle localization

Neu/iPS 19

Neu/iPS 18

C4-dicarboxylate transport - Neu/iPS 6
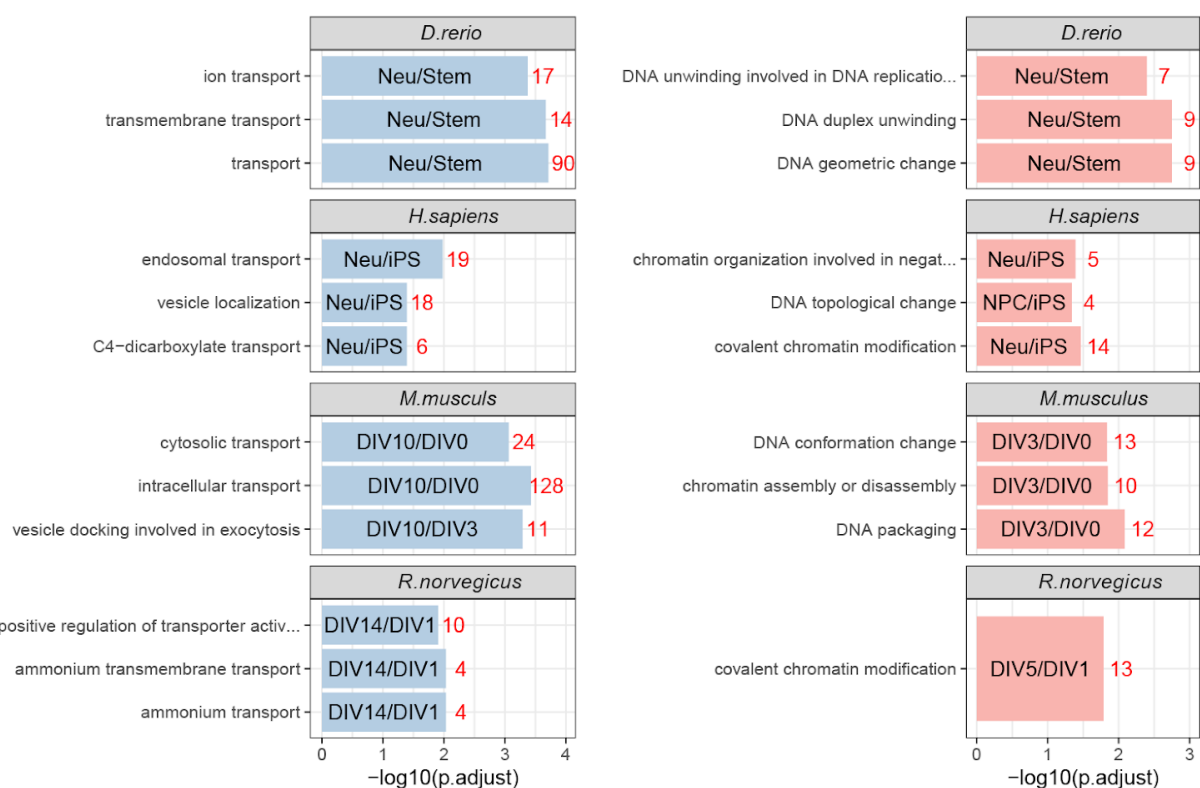

covalent chromatin modification

chromatin assembly or disassembly

DNA packaging

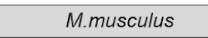

DIV3/DIV0 13 DIV3/DIV0 10

DIV3/DIV0 12

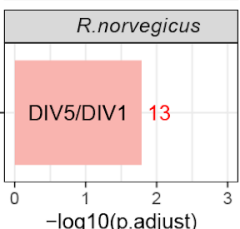

covalent chromatin modification $-\log 10$ (p.adjust) 
bioRxiv preprint doi: https://doi.org/10.1101/2021 07 22.453347: this version posted July 23,2021 . The copyright holder for this preprin (which was not certified by peer review) is the author/funder, who has granted bioRxiv a license to display the preprint in perpetuity. It is made available under aCC-BY-NC-ND 4.0 International license.

\section{Supp.Fig4 - Co-regulation of paralog pairs during neuronal differentiation}

355 A - Scatter plots compare Log2 Fold Change of paralog pairs during neuronal differentiation. Coregulated paralog protein pairs are shown as grey dots, while paralog pairs regulated in opposite directions are shown in orange. A linear regression line is shown in yellow. Only differentially abundant proteins are shown for each dataset. Labels represent the number of unique paralog pairs present in 359 each of the quadrants.

360 B - GO terms enriched among paralog proteins that show opposite regulation during neuronal differentiation (indicated in orange in panel $\mathrm{A}$ ) against the background of all differentially expressed paralog pairs. The pie plots indicate the percentage of differentially regulated paralog annotated with GO terms related to transport, vesicle, and synapses (in blue) and proteins annotated with GO terms related to DNA and chromatin in red. The barplots indicate GO Enrichment (ORA) for the specific GOTerms highlighted in the different pie plots. $x$-axis indicated the -log10(adjusted $p$ value) for the enrichment. The red numbers indicate the amount of significant proteins present in each category. 
A
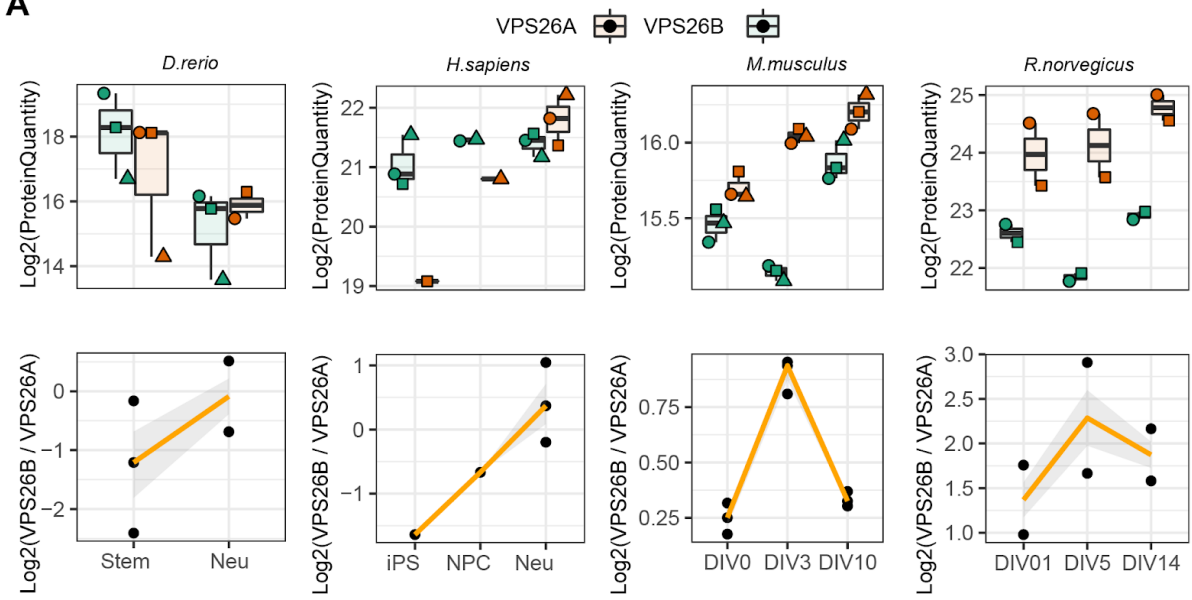

B
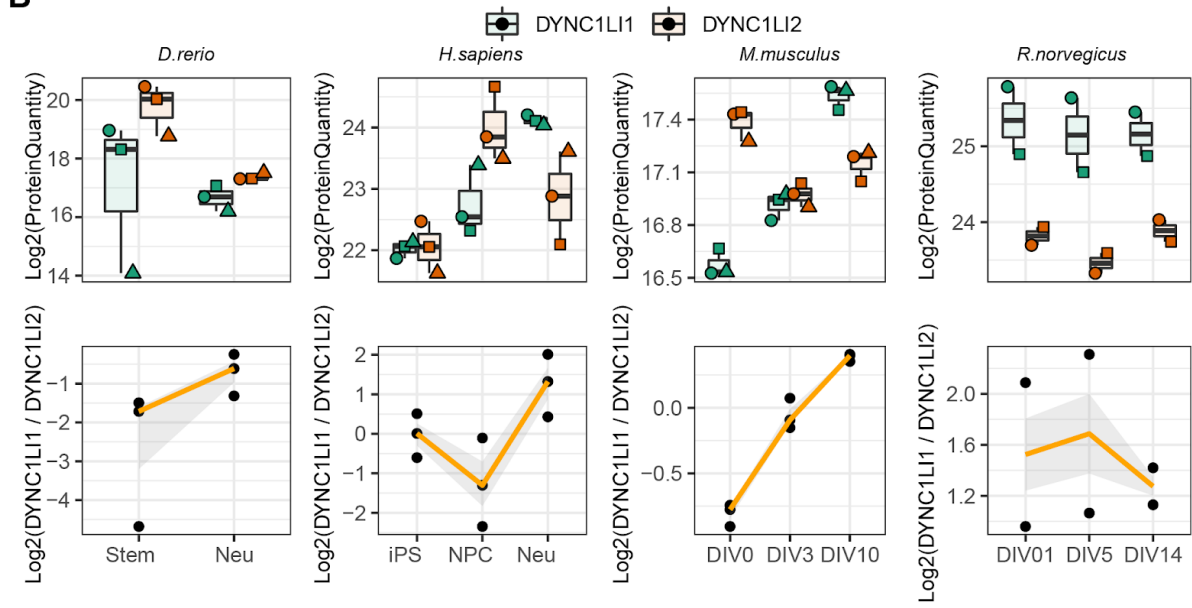

C
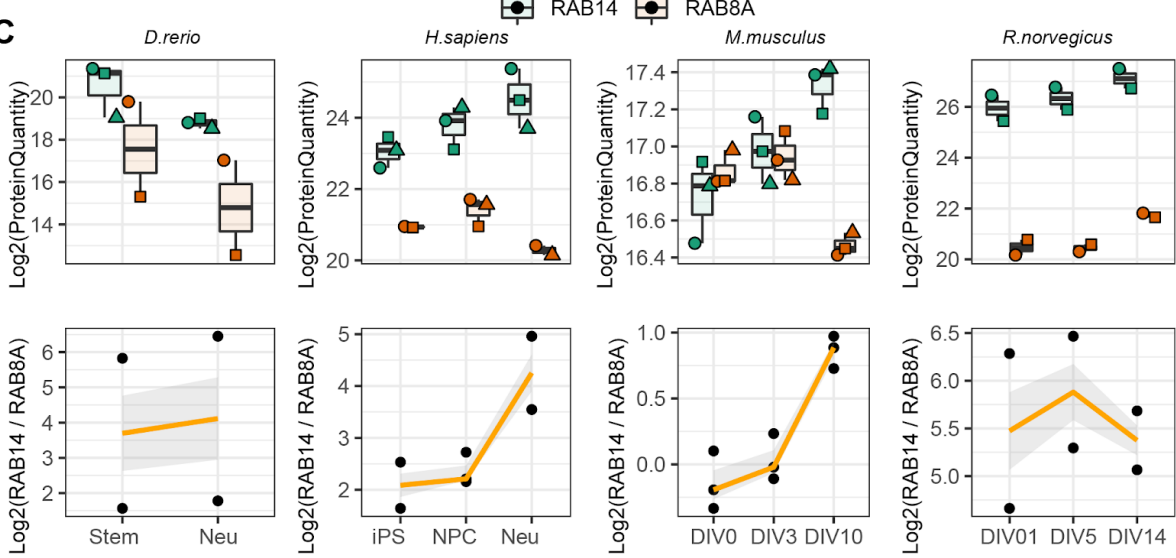

\section{Supp.Fig 5 - Changes of paralog ratios during neuronal differentiation}

A-C - Protein abundance profiles for selected pairs of paralogs across datasets. Boxplots indicate Log2 protein quantities, across different replicates, while line plots (bottom) indicate the ratios between the two paralogs. In the top panel, shapes indicate paired replicate experiments. In the bottom panel, orange lines indicate the mean paralog ratio across replicates, and the shaded area represents $50 \%$ confidence intervals. 

SEC23B. These are highly homologous paralogs that share a high level of protein sequence identity $(>85 \%)$. The potentially divergent functions that these two particular paralogs may have are under debate (Zhu et al. 2015; Khoriaty et al. 2018), however they have never been studied in the context of neuronal differentiation. Using RNA interference (RNAi), we knockeddown either Sec23a or Sec23b in freshly isolated mouse neurons, and analyzed the respective proteome responses during in vitro neuronal differentiation (Fig.4A). First, we confirmed that RNAi significantly reduced the protein abundance of SEC23A relatively to a scrambled siRNA control (Fig4B, Log2 Fold Change siSec23a/siCtrl=-1.42, Qvalue=6.83 E-14, Table S8) and to a lesser extend for SEC23B (Log2 Fold Change siSec23b/siCtrl=-0.42, Qvalue=1.05 E-04, Table S8), globally altering the proportion between SEC23A and SEC23B in the differentiating cells. Interestingly, the knock-down of Sec23a induced a substantial compensatory increase of SEC23B (Log2 Fold Change siSec23a/siCtrl=1.06, Qvalue=6.06 E-10, Table S8), thereby maintaining the total amount of Sec23 (summed abundance of SEC23A and SEC23B) compared to siRNA control (Supp.Fig6A). A similar compensatory increase was true for the knock-down of Sec23b, although to a lesser extent (Fig4B).

In order to understand the impact of an altered balance between the Sec23 paralogs on neuronal differentiation, we compared proteome responses of the different knock downs (KD). The changes in protein abundance caused by the Sec23a-KD or Sec23b-KD were globally correlated when compared to siRNA control $(R=0.52, p<2.2 E-16)$. However, significant paralog-specific differences could be observed (Supp.Fig6B). GO enrichment analysis performed on the direct comparison of SEC23A-KD vs. Sec23b-KD showed that knock down of SEC23B increased the amount of proteins closely related to neuronal activity, i.e., synaptic signalling, whereas, knock down of Sec23a led to an increase of proteins related to DNA replication and RNA transcription (Fig4C, Table S8). Among these proteins, Sec23b knockdown increased the amount of SHC-transforming protein 3 (SHC3), a protein known to promote and regulate axon guidance (Pelicci et al. 2002), SMN1, a component of the Survival Motor Neuron complex, also linked to neurogenesis and neuronal differentiation (Liu et al. 2011; Lauria et al. 2020), and Synaptotagmin-1 (SYT1), a neuronal synaptic protein involved in neurotransmitter release (Coppola et al. 2001) (Fig4D). On the other hand, knock-down of Sec23a increased the expression of the Cyclin-dependent kinases regulatory subunit 2 (CKS2), a protein known to promote cell proliferation (Kang et al. 2009; Lin et al. 2016), and of the transcription factor POU3F3 that has been shown to be necessary for the earliest state of neurogenesis (Sugitani 2002; Dominguez, Ayoub, and Rakic 2013) (Fig4E). This pattern suggests that a higher proportion of SEC23A (as induced by the knockdown of SEC23B) promotes a more 'neuronal' state, while the opposite is true for the SEC23B paralog, which appears to promote a more undifferentiated and proliferative state. In order to investigate whether these responses were more global, we directly compared the effects of Sec23a-KD and Sec23b-KD to the early changes of the proteome that occur between DIV3 and DIV0 using our mouse neuronal differentiation dataset (Supp.Fig3C). The knockdown of Sec23a increased the levels of proteins that are downregulated during neuronal differentiation (Kolmogorov-Smirnov, KS test $\mathrm{p}=3.5 \mathrm{E}-10$, Fig4F, Table S8). In contrast, the knockdown of $\mathrm{Sec} 23 \mathrm{~b}$ promoted an increase of proteins upregulated during neuronal differentiation (Kolmogorov-Smirnov, KS test p.value=7.1E-05, Fig4F, Table S8). This analysis confirms a delaying mouse neuron differentiation in vitro. 
A

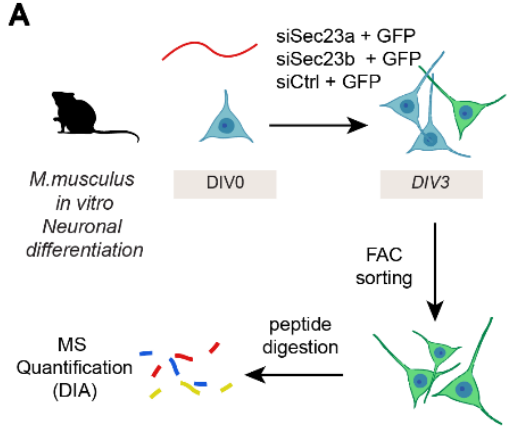

D

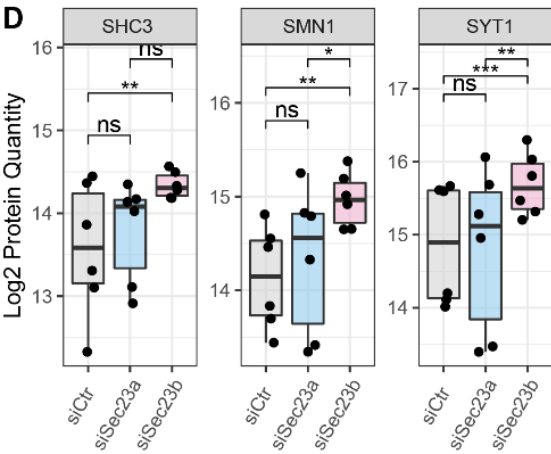

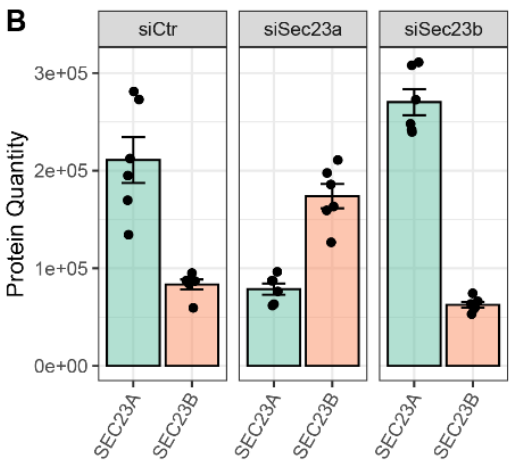
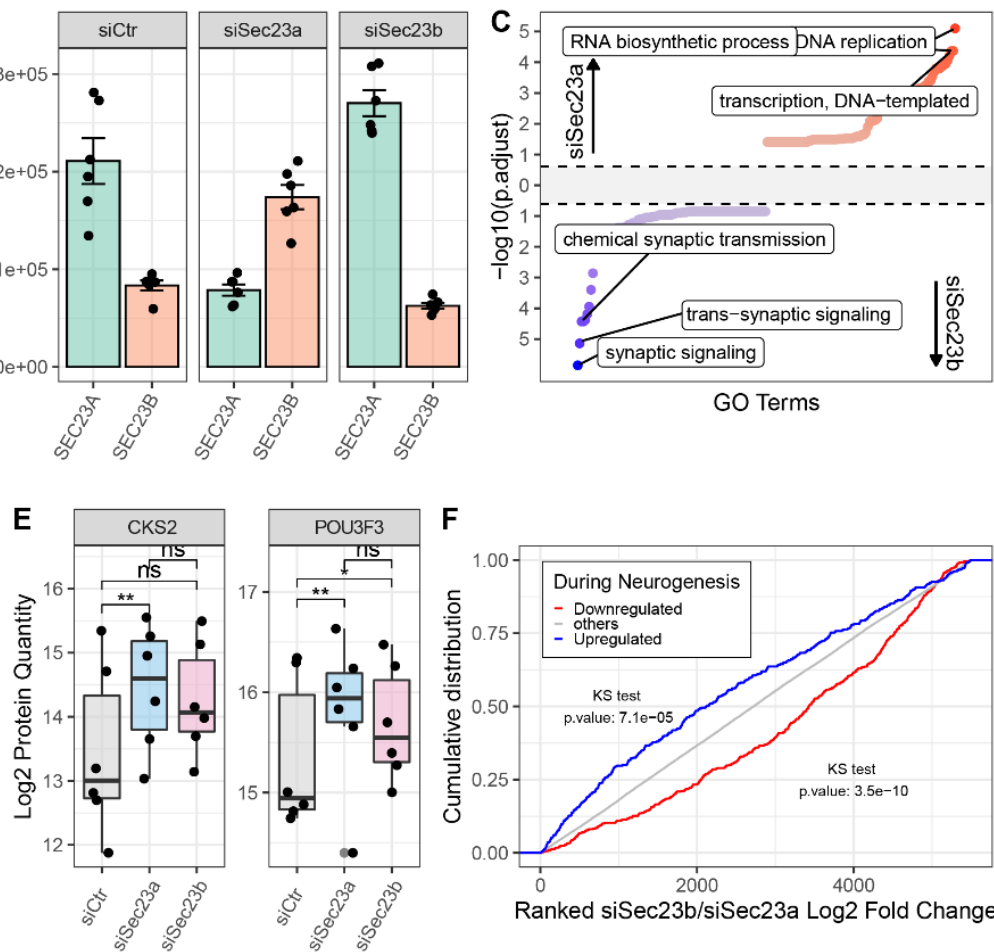

430

431

432

433

434

435

436

437

438

439

440

441

442

443

444

445

446

447

448

449

450
Fig4 - Altering the ratio between SEC23A and SEC23B affects neuronal differentiation in vitro.

A - Mouse cortical neurons isolated from mouse embryos were transfected either with siCtr, siSec23a, or siSec23b, and a GFP expressing plasmid, and differentiated for 3 days. Transfected cells were isolated via FACS based on GFP expression and their proteomes analized by quantitative mass spectrometry (MS) using Data Independent Acquisition (DIA).

$B$ - Protein abundance of SEC23A (green) and SEC23B (orange) following different siRNA treatments, estimated from mass spectrometry data. $n=6$.

C - Gene Set Enrichment Analysis (GSEA) for "Biological Process" category of differentially abundant proteins in siSec23b vs. siSec23a. The $x$ axis represents the $\mathrm{GO}$ terms ranked by their -log10 adjusted $p$ value, for the two conditions, while the y axis represents the -log10(adjusted $p$ value) for each term. Top $100 \mathrm{GO}$ terms enriched among proteins that are more abundant in the siSec23a or siSec23b condition are highlighted in red and blue, respectively.

D, E - Quantification of selected proteins that were differentially affected by siSec23b and siSec23a. Asterisk indicates $p$ values for the indicated comparison, as calculated from mass spectrometry data using Spectronaut (see Methods for details). ${ }^{*} p<=0.05 ;{ }^{* *} p<=0.01,{ }^{* * *} p<=0.001,{ }^{* * * *} p<=0.0001$, ns=not significant. $\mathrm{n}=6$.

F - Cumulative distributions of ranked Log2 fold changes (siSec23b/siSec23a) for proteins that are upregulated (blue) (Log2 FoldChange DIV3/DIV0 $>=1$ and adjusted $p<=0.05$ ), or downregulated (red) (Log2 FoldChange DIV3/DIV0 $<=-1$ and adjusted $p<=0.05$ ) during mouse neuronal differentiation. 

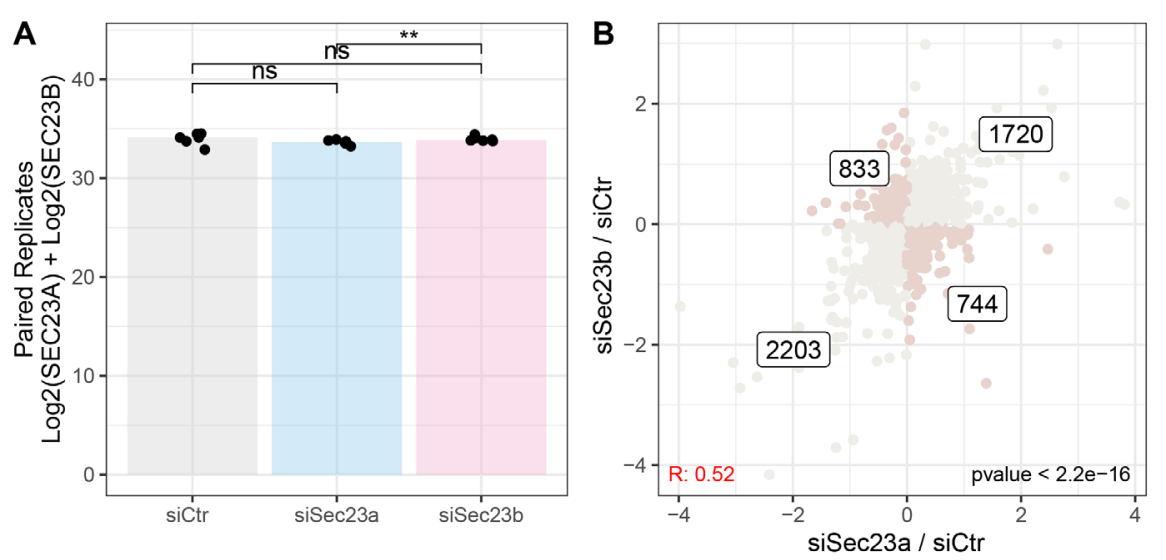

Fig Supp 6 - Effects of SEC23A/B knockdowns

A - Barplot shows the summed Log2 protein quantities of SEC23A and SEC23B following different siRNA treatment. Asterisks indicate significance for the paired two-sample Wilcoxon tests between conditions. ${ }^{* *} p<=0.01$, ns $=$ not significant. $n=6$.

B - Scatterplot showing the relationship between the proteome changes induced by siSec23a ( $x$-axis) and siSec23b ( $y$-axis) against the siCtrl. Proteins affected in a similar way by the knockdown of the paralogs are shown in lightgrey, while the darker dots highlight proteins that are affected in opposite directions. The number of proteins present in each quadrant is indicated. 


\section{Discussion}

In this study, we characterized the specific roles that paralog genes have in promoting transcriptome and proteome variability during development, neuronal differentiation and across different tissues. In accordance with the theory that paralog genes are main carriers of biological variability (Guschanski, Warnefors, and Kaessmann 2017; Ohno 2013), we found that genes that have paralogs are more often differentially expressed across tissues, during development and neuronal differentiation, indicating that they can be used as general descriptors of these specific biological states. New functional modules may then emerge in different cell types by gene duplication and subsequent functional divergence (Arendt et al. 2016; Ori et al. 2016). In agreement with this, we found that divergent expression is particularly pronounced for paralog gene pairs that participate in the formation of protein assemblies. More specifically, the disruption of the relationship between sequence identity and co-expression for this specific group of paralog genes could underline the existence of a specific evolutionary pressure to generate variable "modules" that interchange structurally to form distinct protein complexes with different functions. Consistently, stoichiometrically variable complexes are the ones with the highest paralog content, and they are often associated with functions related to membrane trafficking and chromatin organization. The observed modularity could be then comparable to what has been described for other cellular compartments, such as vertebrate synapses, where gene duplication of scaffold synaptic proteins has been related to the emergence of complex cognitive behaviours (Nithianantharajah et al. 2013).

Similar patterns of paralog regulation were conserved during neuronal differentiation in multiple vertebrates. By comparing data across species, we extracted a specific and conserved paralog exchange signature supporting the hypothesis of module divergence for membrane trafficking-related functions during neuronal differentiation. The relevance of paralog divergence in trafficking complexes has been also recently highlighted by the finding that two members of the COPI complex, COPG1 and COPG2, play distinct roles in modulating mouse neurogenesis (Jain Goyal et al. 2020). This specific substitution was also clearly identified in our mouse data, but not in all other datasets, suggesting that some of these functions could also be species specific. Moreover, we also addressed a similar exchange between the COPII complex members SEC23A and SEC23B that was highly conserved during neuronal differentiation from fish to humans. Previous studies on the functional divergence of these two paralogs reached contradicting conclusions, depending on the model system investigated. Some studies, carried out by substituting SEC23A in the SEC23B gene locus, have proposed a complete functional overlap of these two proteins (Khoriaty et al. 2018). Works by others have indicated separate roles regarding the ability to transport receptors (Scharaw et al. 2016) and cargo substrates (Zhu et al. 2015; Zeng et al. 2015). While these two paralogs are still highly redundant in function, we observed that they carry out different roles in respect to neuronal differentiation, with the SEC23A paralogs being needed to correctly progress during the neuronal differentiation process. Knockdown of either of the two paralogs induced opposite responses during in vitro neuronal differentiation, suggesting that a balanced paralog ratio is needed to correctly modulate this process. 
530 cell types or in different biological states, including pathological ones. Understanding which 531 paralog genes define different cell identities could be exploited in the future for 532 transdifferentiation purposes, e.g., for the generation of new models of neurodegenerative 533 diseases (Mertens et al. 2018). In this case, we can speculate that specific paralog 534 substitutions could help drive lineage transition between different somatic cells. However, 535 broader comparisons between different cell types, integrating multiple data sources, single536 cell analyses, and functional studies of specific paralogs are needed to better elucidate all 537 these different possibilities.

\section{Acknowledgements:}

544 The authors gratefully acknowledge support from the FLI Core Facilities Proteomics, Flow 545 Cytometry, Life Science Computing and Mouse, and the European Molecular Biology 546 Laboratory (EMBL) Proteomics and FACS facilities. The authors thank Ivonne Heinze for processing samples for proteome analysis, Daniela Reichenbach and Christina Valkova for assistance with neuronal mouse culture, and Andrea Gruia for support with fish care. AO

549 acknowledges funding from the German Research Foundation (Deutsche 550 Forschungsgemeinschaft, DFG) via the Research Training Group ProMoAge (GRK 2155), the Else Kröner Fresenius Stiftung (award number: 2019_A79), the Fritz-Thyssen foundation (award number: 10.20.1.022MN) and the Chan Zuckerberg Initiative Neurodegeneration Challenge Network (NDCN). The FLI is a member of the Leibniz Association and is financially supported by the Federal Government of Germany and the State of Thuringia. CK acknowledges funding from the German Research Foundation (Deutsche Forschungsgemeinschaft, DFG, grant number KA 1751/8-1). MHC acknowledges funding from Fondazione AIRC per la Ricerca sul Cancro (AIRC, [IG 23539 to M.H.C.] ).

\section{Author contributions}

Conceptualization: DDF, LP, MB, AO. Data curation: DDF, LP. Experimental procedures: MA, MTM, LB, AAP, AO. Methodology: MA, MTM, AAP, AO. Project administration: AO, MB. Data analysis: DDF, LP. Supervision: AO, MB, CK, MHC, DG. Visualization: DDF. Writing - original draft: DDF, AO. Writing - review \& editing: MA, MTM, LP, LB, MHC, CK, MB.

\section{Conflict of interest}

564 The authors declare no conflict of interest. 
$\rightarrow$ Table S1 - Zebrafish development data (White et al., 2017) and proteome and transcriptome data (Wang et al., 2019) across human tissues. This table also contains paralog pairs correlation values for those datasets.

$\rightarrow$ Table S2 - Protein complex and subunits co-expression during zebrafish development and across human tissues.

$\rightarrow$ Table S3 - GO Enrichment Analysis of variable subunit pairs during zebrafish development and across human tissues.

$\rightarrow$ Table S4 - Global proteomics data for neuronal differentiation in Mouse, Human, Rat and Zebrafish.

$\rightarrow$ Table S5 - Protein quantification data for paralog protein pairs during neuronal differentiation.

$\rightarrow$ Table S6 - Protein complex subunits co-expression during neuronal differentiation.

$\rightarrow$ Table 57 - Paralog pairs ratios during neuronal differentiation across species.

589

$\rightarrow$ Table S8 - Global proteomics data following SEC23A and SEC23B knockdowns

590 during mouse neuronal differentiation.

591

592

593

594

595

596

597

598

599

600

601

602

603

604

605

606

607

608

609

610

611

612

613 


\section{Materials and Methods}

615

\section{Dataset and Resources}

\section{Ensembl Compara paralog genes resources}

618 Paralogs annotation for Homo sapiens (GRch38.p13) Danio rerio (GRCz11)

619 Mus musculus (GRCm38.p6) Rattus norvegicus (Rnor_6.0), were downloaded from Ensembl

620 (v102) via biomart

621 (http://www.ensembl.org/biomart/martview/f04b3aa8b5c7f463e3edf9fa58d205a7).

622 Duplicated paralog pairs ( e.g, Paralog1 | Paralog2 ; Paralog2 | Paralog1) were removed from 623 each dataset, so that only unique pairs (Paralog1 | Paralog2) were retained.

624

625

\section{Protein Complexes Resources}

626 Protein Complexes definition were taken from (Ori et al., 2016). Members of protein complexes were mapped by orthology in Danio rerio and Rattus Norvegicus using the bioconductor

628 package 'biomaRt' (Durinck et al. 2009) using as reference the Homo sapiens protein

629

630

631 complexes definitions.

632

\section{Publicly Available Data used in this study}

633

Zebrafish Embryo development data were obtained from White et al., 2017. (Supplementary

634 file 3). Human Proteome and Transcriptome data across tissues were obtained from Wang et al., 2019 (Table EV2). Protein identification and LFQ intensity values (Log2) in cultured human iPSCs, NPCs and differentiated neurons, were obtained from Supplementary table S2 from (Djuric et al. 2017). Rat neuronal differentiation data published in Frese et al., 2017, were

638 (http://proteomecentral.proteomexchange.org/cgi/GetDataset?ID=PXD005031) and analyzed again as described below.

\section{Isolation of embryonic stem cells and neurons from Zebrafish}

641 Zebrafish (Danio rerio) strains were maintained following standard protocols (Westerfield, 642 2007) in the Gilmour lab at the EMBL, Heidelberg, Germany. Embryos were raised in E3 buffer $643(5 \mathrm{mM} \mathrm{NaCl}, 0.17 \mathrm{mM} \mathrm{KCl}, 0.33 \mathrm{mM} \mathrm{CaCl} 2,0.33 \mathrm{mM} \mathrm{MgSO} 4)$ at 26 to $30^{\circ} \mathrm{C}$. All zebrafish 644 experiments were conducted on embryos younger than $3 \mathrm{dpf}$. For isolation of undifferentiated 645 cells a wild type strain (golden) and for neuronal cells the NBT-DsRed strain were used (Peri 646 and Nüsslein-Volhard 2008).

647

\section{Early embryos (6 hours post fertilization (hpf))}

650 Wild type embryos were removed from their chorions using $1 \mathrm{ml}$ of pronase (stock $30 \mathrm{mg} / \mathrm{ml}$ ) in $40 \mathrm{ml}$ buffer E3 and incubated for 10-15 min with gentle shaking every 2 min in a small beaker. The supernatant was removed and the embryos were washed $4-5$ times using buffer E3. The embryos were splitted into batches of around 250-300 per $1.5 \mathrm{ml}$ tube. $1 \mathrm{ml}$ of deyolking buffer ( $55 \mathrm{mM} \mathrm{NaCl}, 1.8 \mathrm{mM} \mathrm{KCl}, 1.25 \mathrm{mM} \mathrm{NaHCO})$ was added per tube and everything passed twice through a $200 \mu$ l pipet tip. The tubes were incubated at RT in a shaker at $1100 \mathrm{rpm}$ for $5 \mathrm{~min}$ and afterwards spun at $300 \mathrm{x} \mathrm{g}$ for $30 \mathrm{sec}$ to remove the supernatant. The embryos were washed using $1 \mathrm{ml}$ of wash buffer $(110 \mathrm{mM} \mathrm{NaCl}, 3.5 \mathrm{mM} \mathrm{KCl}, 2.7 \mathrm{mM}$ $\mathrm{CaCl} 2$ and $10 \mathrm{mM}$ Tris/HCl pH 8.5), shaken at $1100 \mathrm{rpm}$ at RT for $2 \mathrm{~min}$ and spun as above to remove the supernatant. The wash step was repeated twice. The deyolked and dissociated 
659

660

661

662

663

664

665

666

667

668

669

670

671

672

673

674

675

676

677

678

679

680

681

682

683

684

685

686

687

688

689

690

691

692

693

694

695

696

697

698

699

700

701

702

703

704

705

embryos were resuspended in $400 \mu \mathrm{l}$ wash buffer and passed through a $40 \mu \mathrm{m}$ cell strainer to remove undissociated cells. The merged cells were washed as above and resuspended in $110 \mu \mathrm{I}$ PBS and counted using a hemocytometer.

\section{Late embryos (24 hpf)}

After $24 \mathrm{hpf}$, the NBT dsRed positive embryos were manually sorted. Up to the addition of the deyolking buffer all steps were the same as for early embryos. After the addition of $1 \mathrm{ml}$ deyolking buffer per tube, the embryos were passed 10 times through a $1000 \mu \mathrm{l}$ pipet tip, followed by washing twice with deyolking buffer and four times with washing buffer. For better cell dissociation the embryos were rinsed once with Accumax (Millipore) and then resuspended in $1 \mathrm{ml}$ Accumax and transferred to a $15 \mathrm{ml}$ tube. The embryos were incubated at RT for $5 \mathrm{~min}$ at the lowest speed of the vortex mixer. The embryos were dissociated by pipetting for 2 min using a $1000 \mu$ pipet tip, 2 min incubation on the vortex mixer and 1 min of additional pipetting. The cells were spun for $1 \mathrm{~min}$ at $300 \mathrm{x} g$ at RT and washed twice using 1 $\mathrm{ml}$ of PBS with $0.5 \%$ BSA. $400 \mu \mathrm{l}$ of PBS with $0.5 \%$ BSA was used per tube to resuspend the cells afterwards passed through a $40 \mu \mathrm{m}$ cell strainer and merged. DNAse I (Roche, 10 $\mathrm{mg} / \mathrm{ml}$ in water) $170 \mathrm{U} / \mathrm{ml}$ and $10 \mathrm{mM} \mathrm{MgCl} 2$ was added. Cells expressing the DsRed fluorescent protein were FAC sorted with a MoFlo cell sorter (Beckman Coulter $\mathrm{GmbH}$, Krefeld, Germany) to obtain a highly enriched fraction for neuronal cells.

\section{In vitro differentiation of mouse cortical neurons}

\section{Animal management practices}

All mice were maintained in specific pathogen-free conditions, with food and water available ad libitum. The animal room had a constant temperature of $21^{\circ} \mathrm{C} \pm 2,55 \% \pm 15$ humidity, and controlled lighting (12 $\mathrm{h}$ light/dark cycle). The location for animal keeping was animal house TH4 at Leibniz Institute on Aging (Fritz Lipmann Institute), Jena, Germany. Breeding was license-free and performed under $\$ 11$ TierSchG. Euthanasia and organ removal were performed under the internal §4 TierSchG licences O_CK_18-20 and O-CK_21-23. Euthanasia of mice was performed in a chamber with controlled $\mathrm{CO} 2$ fill rate according to "Directive 2010/63/EU annex IV of the European Parliament and the Council on the protection of animals used for specific purposes".

\section{Mouse neuronal cell culture}

Cortical neurons were isolated from wild type murine embryonic brains (E15.5) of mixed background (FVB/NJ, C57BL/6, 129/Sv) and differentiated in glia-conditioned neurobasal medium. Briefly, meninges were removed, cortices were isolated, minced and dissociated in trypsin EDTA (Invitrogen), solution for $15 \mathrm{~min}$ at $37^{\circ} \mathrm{C}$. The supernatant was removed and the tissue was washed 3 times with trituration solution (10 mM HEPES, $1 \%$ penicillin/streptomycin, $10 \mathrm{mM}$ L-glutamine, 1\% BSA, 10\% FBS, 0.008\% DNase in HBSS) and homogenized in trituration solution using fire polished glass pipettes. For the mouse in vitro neuronal differentiation data, neurons were counted and pellets containing 1 million cells (DIV0) were prepared and frozen until further use. Additionally, 1 million cells were seeded on poly-L-lysine coated $6 \mathrm{~cm}$ plates containing $4 \mathrm{ml}$ glia-conditioned plating medium (1\% penicillin/streptomycin, $1 \mathrm{mM}$ sodium pyruvate, $0.5 \%$ glucose, $10 \mathrm{mM}$ HEPES 1x B27 supplement, $10 \%$ FBS, $10 \mathrm{mM} \mathrm{L-glutamine}$ in MEM). After $24 \mathrm{~h}$ the plating medium was substituted by glia-conditioned neurobasal medium (10 mM HEPES, 1x B27 supplement, 5 
mM L-glutamine in NBM). Neurons were collected at DIV3 and DIV10. To this end, neurons were scraped off in cold PBS, obtained cell suspensions were transferred to a microcentrifuge tube and centrifuged for $5 \mathrm{~min}$ at $4{ }^{\circ} \mathrm{C}$ and $500 \mathrm{~g}$. The obtained pellets were washed with PBS twice and frozen until further use.

710 For preparation of glia-conditioned mediums, a primary astroglia culture was established. For this purpose, brains were isolated from 15.5 days old embryos, the meninges were removed, the cerebral hemispheres were minced and afterwards dissociated in trypsin solution for 15 min at $37^{\circ} \mathrm{C}$. Finally, the tissue was homogenized by pipetting and cells were plated on a 10 $\mathrm{cm}$ dish containing glia medium (1\% penicillin/streptomycin, $1 \mathrm{mM}$ sodium pyruvate, $0.5 \%$ glucose, $10 \mathrm{mM}$ HEPES, $20 \mathrm{mM}$ L-glutamine, 10\% FBS in MEM) and grown to confluence. For preconditioning of neurobasal medium or plating medium, the media were added to the glia feeder cultures and collected after $24 \mathrm{~h}$.

\section{SEC23A and SEC23B knockdown in mouse neuronal differentiation}

For the Sec23 paralogs knockdowns, Cortical neurons were isolated from C57BL/6JRj mouse embryo (Janvier), as described above. Then, freshly isolated neurons (5 million cells per nucleofection reaction) were transfected using the $4 \mathrm{D}$-Nucleofector ${ }^{\mathrm{TM}} \mathrm{X}$ Unit and the P3 Primary Cell 4D Nucleofector $X$ kit (Lonza, Switzerland), as indicated. Cells were transfected with $250 \mathrm{nM}$ of siRNA and $1 \mu \mathrm{l}$ of control pMax GFP (Nucleofector X kit, Lonza), using the CU133 program. Immediately after transfection, cells were plated on poly-L-lysine (SigmaAldrich) - coated $10 \mathrm{~cm}$ plates containing $10 \mathrm{ml}$ of glia-conditioned plating medium: $1 \%$ penicillin/streptomycin, $1 \mathrm{mM}$ sodium pyruvate (Sigma- Aldrich), 0.5\% glucose, $10 \mathrm{mM}$ HEPES, 1x B27 supplement (Invitrogen), 10\% FBS, $10 \mathrm{mM}$ L-glutamine in MEM (Invitrogen $31095-052$ ), and incubated at $37^{\circ} \mathrm{C}$. After 1 day, the medium was replaced with gliaconditioned neurobasal medium: $10 \mathrm{mM}$ HEPES, 1x B27 supplement, $5 \mathrm{mM}$ L-glutamine in NBM (Invitrogen). After 3 days in culture, neurons were washed twice with PBS, detached using Trypsin EDTA (3-5 min, $37^{\circ} \mathrm{C}$ ), collected in $5 \mathrm{ml}$ of PBS with $2 \% \mathrm{FBS}$, and pelleted by centrifugation ( $450 \mathrm{~g}, 8 \mathrm{~min}$, room temperature). Pellets were resuspended in $0.3 \mathrm{ml}$ PBS with $2 \%$ FBS, and GFP positive cells were labeled with Sytox Blue Dead Cell Stain (viable staining) (Molecular Probes, ThermoFisher Scientific) and sorted directly in $200 \mu \mathrm{l}$ of $2 x$ lysis buffer (200 mM HEPES pH 8.0,100 mM DTT, 4\% SDS) using a BD FACSAria Fusion with the Software BD FACSDiva 8.0.1 and 9.0.1 (BD Biosciences), using $488 \mathrm{~nm}$ laser and 530/30 filter for the GFP signal and laser $405 \mathrm{~nm}$ and $450 / 50$ filter for the Sytox blue.

\section{Sample preparation for Mass Spectrometry}

\section{Sample preparation and dimethyl labeling for Zebrafish stem cells and neurons} and $4 \mathrm{M}$, respectively, and sonicated for $3 \times 30 \mathrm{sec}$ to shear chromatin. Before protein digestion, samples were stored at $-80^{\circ} \mathrm{C}$. Samples were quickly thawed and sonicated for 1 min. DTT was added to a final concentration of $10 \mathrm{mM}$ and incubated for 30 min with mixing at $800 \mathrm{rpm}$ to reduce cysteines. Then $15 \mathrm{mM}$ of freshly prepared iodoacetamide (IAA) was added and samples were incubated for $30 \mathrm{~min}$ at room temperature in the dark to alkylate cysteines. Afterwards, 1:100 (w/w) LysC (Wako Chemicals $\mathrm{GmbH}$ ) was added for $4 \mathrm{~h}$ at $37^{\circ} \mathrm{C}$ with mixing at $800 \mathrm{rpm}$. Then urea concentration was diluted to $1.5 \mathrm{M}$ with HPLC water and 
1:50 (w/w) trypsin (Promega $\mathrm{GmbH}$ ) was added for $12 \mathrm{~h}$ at $37{ }^{\circ} \mathrm{C}$ with mixing at $700 \mathrm{rpm}$. Afterwards the samples were acidified with $10 \%$ TFA and the cleavage of Rapigest was allowed to proceed for $30 \mathrm{~min}$ at $37^{\circ} \mathrm{C}$. After spinning the sample for $5 \mathrm{~min}$ at 13,000x $\mathrm{g}$ at room temperature the supernatant was transferred to a new tube to proceed with peptide desalting.

For desalting and cleaning-up of the digested sample, C-18 spin columns (Sep-Pak C18 Classic Cartridge, Waters) were used. A vacuum manifold was used for all washing and elution steps. First the columns were equilibrated with $100 \%$ methanol and then washed twice with $5 \%(\mathrm{v} / \mathrm{v})$ acetonitrile (ACN) and $0.1 \%(\mathrm{v} / \mathrm{v})$ formic acid (FA). The sample was loaded two times and then the column was washed 2 times with $5 \%(\mathrm{v} / \mathrm{v}) \mathrm{ACN}$ and $0.1 \%(\mathrm{v} / \mathrm{v}) \mathrm{FA}$. The undifferentiated cell samples were labeled using a 'light' labeling reagent and the FACS sorted neuronal cells were labeled using an 'intermediate' labeling reagent inducing a mass shift of 28 or 32 Da respectively (Boersema et al. 2009) . Formaldehyde and the D-isotopomer of formaldehyde react with primary amines of peptides ( $\mathrm{N}$-terminus and side chains of lysines) and generate a mass shift of $4 \mathrm{Da}$. The labeling reagents consisted of $4.5 \mathrm{ml} 50 \mathrm{mM}$ sodium phosphate buffer (mixture of $100 \mathrm{mM} \mathrm{NaH2PO} 4$ and $100 \mathrm{mM} \mathrm{Na2HPO4),} \mathrm{pH} \mathrm{7.5,} 250 \mu \mathrm{l} 600$ $\mathrm{mM} \mathrm{NaBH} 3 \mathrm{CN}$ and $250 \mu \mathrm{l} 4 \%$ formaldehyde for light or $4 \%$ deuterated formaldehyde for intermediate labeling reagent, per sample. After the labeling procedure, the column was washed 2 times with $5 \%(\mathrm{v} / \mathrm{v}) \mathrm{ACN}$ and $0.1 \%(\mathrm{v} / \mathrm{v})$ FA. For elution $50 \%(\mathrm{v} / \mathrm{v}) \mathrm{ACN}$ and $0.1 \%$ (v/v) FA was used. Labelled peptides from undifferentiated cells and FACS sorted neurons were pooled, dried in a vacuum concentrator, and resuspended in $20 \mathrm{mM}$ ammonium formate ( $\mathrm{pH}$ 10.0), to be ready for high $\mathrm{pH}$ reverse-phase peptide fractionation. To dissolve the dried samples, they were vortexed, mixed for $5 \mathrm{~min}$ at maximum speed in a thermomixer and sonicated for $90 \mathrm{~s}$. The samples were stored at $-20^{\circ} \mathrm{C}$.

\section{High $\mathrm{pH}$ reverse-phase peptide fractionation for dimethyl labelled samples}

Offline high pH reverse-phase fractionation was performed using an Agilent 1200 Infinity HPLC System equipped with a quaternary pump, degasser, variable wavelength UV detector (set to $254 \mathrm{~nm}$ ), peltier-cooled autosampler, and fraction collector (both set at $10^{\circ} \mathrm{C}$ ). The column was a Gemini C18 column ( $3 \mu \mathrm{m}, 110 \AA, 100 \times 1.0 \mathrm{~mm}$, Phenomenex) with a Gemini C18, $4 \times 2.0 \mathrm{~mm}$ SecurityGuard (Phenomenex) cartridge as a guard column. The solvent system consisted of $20 \mathrm{mM}$ ammonium formate $(\mathrm{pH} \mathrm{10.0)}$ as mobile phase $\mathrm{A}$ and $100 \%$ acetonitrile as mobile phase $B$. The separation was accomplished at a mobile phase flow rate of $0.1 \mathrm{ml} / \mathrm{min}$ using the following linear gradient: $99 \%$ A for $2 \mathrm{~min}$, from $99 \% \mathrm{~A}$ to $37.5 \% \mathrm{~B}$ in $61 \mathrm{~min}$, to $85 \% \mathrm{~B}$ in a further $1 \mathrm{~min}$, and held at $85 \% \mathrm{~B}$ for an additional $5 \mathrm{~min}$, before returning to $99 \% \mathrm{~A}$ and re-equilibration for $18 \mathrm{~min}$. Thirty two fractions were collected along with the LC separation that were subsequently pooled into 10 fractions. Pooled fractions were dried in a speed-vac and resuspended in $5 \%(\mathrm{v} / \mathrm{v}) \mathrm{ACN}$ and $0.1 \%(\mathrm{v} / \mathrm{v}) \mathrm{FA}$ and then stored at $-80{ }^{\circ} \mathrm{C}$ until LC-MS/MS analysis.

\section{Sample preparation for in vitro differentiated mouse neurons}

Frozen cell pellets of in vitro differentiated mouse neurons ( 1 million cells per sample) were thawed and resuspended in $100 \mu \mathrm{l}$ of $1 x$ PBS. An equivalent amount of 2x Lysis Buffer (200 $\mathrm{mM}$ HEPES pH8.0, $100 \mathrm{mM}$ DTT, 4\% SDS) was added to the lysate, for a total volume of $200 \mu \mathrm{l}$. For neurons treated with SEC23A/b or control siRNA, cells (between 40,000 and 
801 high intensity at $20^{\circ} \mathrm{C}$. Samples were then boiled for $10 \mathrm{~min}$ at $95^{\circ} \mathrm{C}$, and a second sonication 802 cycle was performed as described above. The lysates were centrifuged at 18,407x $\mathrm{g}$ for $1 \mathrm{~min}$. 803 Subsequently, samples were reduced using $10 \mathrm{mM}$ DTT for $15 \mathrm{~min}$ at $45^{\circ} \mathrm{C}$, and alkylated 804 using freshly made $15 \mathrm{mM}$ IAA for $30 \mathrm{~min}$ at room temperature in the dark. Subsequently, proteins were precipitated using acetone and digested using LysC (Wako sequencing grade) and trypsin (Promega sequencing grade), as described in (Buczak et al. 2020). The digested proteins were then acidified with $10 \%(\mathrm{v} / \mathrm{v})$ trifluoroacetic acid. The eluates were dried down using a vacuum concentrator, and reconstituted samples in $5 \%(\mathrm{v} / \mathrm{v})$ acetonitrile, $0.1 \%(\mathrm{v} / \mathrm{v})$ formic acid. For Data Independent Acquisition (DIA) based analysis (siRNA treated neurons), samples were transferred directly to an MS vial, diluted to a concentration of $1 \mu \mathrm{g} / \mu \mathrm{l}$, and spiked with iRT kit peptides (Biognosys, Zurich, Switzerland) prior to analysis by LC-MS/MS. For Tandem Mass Tags (TMT) based analysis (time course of in vitro differentiation), samples were further processed for TMT labelling as described below.

\section{TMT labelling and high $\mathrm{pH}$ reverse-phase peptide fractionation}

Following desalting, peptides were dried in a vacuum concentrator and buffered using $0.1 \mathrm{M}$ HEPES buffer $\mathrm{pH} 8.5$ (1:1 ratio) for labelling, and then sonicated in a Bioruptor Plus for 5 cycles with $1 \mathrm{~min}$ ON and $30 \mathrm{~s}$ OFF with high intensity. 10-20 $\mu \mathrm{g}$ peptides were taken for each labelling reaction. TMT-10plex reagents (Thermo Scientific, Waltham, MA, USA) labeling was performed by addition of $1 \mu \mathrm{l}$ of the TMT reagent. After $30 \mathrm{~min}$ of incubation at room temperature with shaking at $600 \mathrm{rpm}$ in a thermomixer (Eppendorf, Hamburg, Germany), a second portion of TMT reagent $(1 \mu \mathrm{l})$ was added and incubated for another $30 \mathrm{~min}$. After checking labelling efficiency, samples were pooled, desalted with Oasis ${ }^{\circledR}$ HLB $\mu$ Elution Plate and subjected to high $\mathrm{pH}$ fractionation prior to $\mathrm{MS}$ analysis. Offline high $\mathrm{pH}$ reverse phase fractionation was performed using a Waters XBridge C18 column $(3.5 \mu \mathrm{m}, 100 \times 1.0 \mathrm{~mm}$, Waters) with a Gemini C18, $4 \times 2.0 \mathrm{~mm}$ SecurityGuard (Phenomenex) cartridge as a guard column on an Agilent 1260 Infinity HPLC, as described in (Buczak et al. 2020). Forty-eight fractions were collected along with the LC separation, which were subsequently pooled into 16 fractions. Pooled fractions were dried in a vacuum concentrator and then stored at $-80^{\circ} \mathrm{C}$ until LC-MS/MS analysis.

\section{Mass Spectrometry data acquisition}

\section{Data Dependent Acquisition for dimethyl labelled samples (Zebrafish neurons and stem cells)}

838 The 10 fractions obtained by high $\mathrm{pH}$ fractionation were analyzed using a nanoAcquity UPLC system (Waters $\mathrm{GmbH}$ ) connected online to a LTQ-Orbitrap Velos Pro instrument (Thermo Fisher Scientific $\mathrm{GmbH})$. Peptides were separated on a BEH300 C18 (75 $\mu \mathrm{m}$ x $250 \mathrm{~mm}, 1.7$ $\mu \mathrm{m})$ nanoAcquity UPLC column (Waters $\mathrm{GmbH}$ ) using a stepwise 145 min gradient between 3 and $85 \%(\mathrm{v} / \mathrm{v}) \mathrm{ACN}$ in $0.1 \%(\mathrm{v} / \mathrm{v})$ FA. Data acquisition was performed using a TOP-20 strategy where survey MS scans ( $\mathrm{m} / \mathrm{z}$ range $375-1600)$ were acquired in the orbitrap $(R=30,000 \mathrm{FWHM})$ and up to 20 of the most abundant ions per full scan were fragmented by collision-induced dissociation (normalized collision energy $=35$, activation $Q=0.250$ ) and analyzed in the LTQ. Ion target values were 1 e6 (or 500 ms maximum fill time) for full scans and $1 \mathrm{e} 5$ (or $50 \mathrm{~ms}$ maximum fill time) for MS/MS scans. Charge states 1 and unknown were 
848 rejected. Dynamic exclusion was enabled with repeat count=1, exclusion duration=60 s, list size $=500$ and mass window $\pm 15 \mathrm{ppm}$. The 16 fractions obtained by high $\mathrm{pH}$ fractionation were resuspended in $10 \mu \mathrm{L}$ reconstitution buffer (5\% (v/v) acetonitrile, 0.1\% (v/v) TFA in water) and $3 \mu \mathrm{L}$ were injected. Peptides were separated using the nanoAcquity UPLC system (Waters) fitted with a trapping (nanoAcquity Symmetry C18, $5 \mu \mathrm{m}, 180 \mu \mathrm{m} \times 20 \mathrm{~mm}$ ) and an analytical column (nanoAcquity BEH C18, 2.5 $\mu \mathrm{m}, 75 \mu \mathrm{m} \times 250 \mathrm{~mm}$ ). The outlet of the analytical column was coupled directly to an Orbitrap Fusion Lumos (Thermo Fisher Scientific) using the Proxeon nanospray source. Solvent A was 858 water, $0.1 \%(\mathrm{v} / \mathrm{v})$ formic acid, and solvent $\mathrm{B}$ was acetonitrile, $0.1 \%(\mathrm{v} / \mathrm{v})$ formic acid. The samples were loaded with a constant flow of solvent $A$ at $5 \mu \mathrm{L} / \mathrm{min}$, onto the trapping column. Trapping time was $6 \mathrm{~min}$. Peptides were eluted via the analytical column at a constant flow of $0.3 \mu \mathrm{L} / \mathrm{min}$, at $40{ }^{\circ} \mathrm{C}$. reconstitution buffer $(5 \%(\mathrm{v} / \mathrm{v})$ acetonitrile, $0.1 \%(\mathrm{v} / \mathrm{v})$ TFA in water) and $3.5 \mu \mathrm{L}$ were injected. Peptides were eluted using a linear gradient from 5 to $7 \%$ in $10 \mathrm{~min}$, then from $7 \% \mathrm{~B}$ to $28 \% \mathrm{~B}$ in a further $105 \mathrm{~min}$ and to $45 \% \mathrm{~B}$ by $120 \mathrm{~min}$. The peptides were introduced into the mass spectrometer via a Pico-Tip Emitter $360 \mu \mathrm{m}$ OD $\times 20 \mu \mathrm{m}$ ID; $10 \mu \mathrm{m}$ tip (New Objective), and a spray voltage of $2.2 \mathrm{kV}$ was applied. The capillary temperature was set at $300{ }^{\circ} \mathrm{C}$. Full-scan MS spectra with mass range $375-1500 \mathrm{~m} / \mathrm{z}$ were acquired in profile mode in the Orbitrap with resolution of $60,000 \mathrm{FWHM}$ using the quad isolation. The RF on the ion funnel was set to $40 \%$. The filling time was set at a maximum of $100 \mathrm{~ms}$ with an AGC target of $4 \times 105$ ions and 1 microscan. The peptide monoisotopic precursor selection was enabled along with relaxed restrictions if too few precursors were found. The most intense ions (instrument operated for a 3 s cycle time) from the full scan MS were selected for MS2, using quadrupole isolation and a window of $1 \mathrm{Da}$. HCD was performed with collision energy of $35 \%$. A maximum fill time of $50 \mathrm{~ms}$ for each precursor ion was set. MS2 data were acquired with a fixed first mass of $120 \mathrm{~m} / \mathrm{z}$. The dynamic exclusion list was with a maximum retention period of $60 \mathrm{~s}$ and relative mass window of $10 \mathrm{ppm}$. For the MS3, the precursor selection window was set to the range $400-2000 \mathrm{~m} / \mathrm{z}$, with an exclude width of $18 \mathrm{~m} / \mathrm{z}$ (high) and $5 \mathrm{~m} / \mathrm{z}$ (low). The most intense fragments from the MS2 experiment were co-isolated (using Synchronus Precursor Selection=8) and fragmented using HCD (65\%). MS3 spectra were acquired in the Orbitrap over the mass range $100-1000 \mathrm{~m} / \mathrm{z}$ and resolution set to $30,000 \mathrm{FWMH}$. The maximum injection time was set to $105 \mathrm{~ms}$, and the instrument was set not to injections for all available parallelizable time.

\section{Data Independent Acquisition (SEC23A/b knockdowns)}

Peptides were separated in trap/elute mode using the nanoAcquity MClass Ultra-High Performance Liquid Chromatography system (Waters, Waters Corporation, Milford, MA, USA) equipped with a trapping (nanoAcquity Symmetry C18, $5 \mu \mathrm{m}, 180 \mu \mathrm{m} \times 20 \mathrm{~mm}$ ) and an analytical column (nanoAcquity BEH C18, $1.7 \mu \mathrm{m}, 75 \mu \mathrm{m} \times 250 \mathrm{~mm}$ ). Solvent $A$ was water and $0.1 \%$ formic acid, and solvent $B$ was acetonitrile and $0.1 \%$ formic acid. $1 \mu$ of the samples ( $\square 1 \mu \mathrm{g}$ on column) were loaded with a constant flow of solvent $A$ at $5 \mu \mathrm{l} / \mathrm{min}$ onto the trapping column. Trapping time was $6 \mathrm{~min}$. Peptides were eluted via the analytical column with a constant flow of $0.3 \mu \mathrm{l} / \mathrm{min}$. During the elution, the percentage of solvent $\mathrm{B}$ increased in a nonlinear fashion from $0-40 \%$ in $120 \mathrm{~min}$. Total run time was $145 \mathrm{~min}$. including equilibration and conditioning. The LC was coupled to an Orbitrap Exploris 480 (Thermo Fisher Scientific, Bremen, Germany) using the Proxeon nanospray source. The peptides were introduced into the mass spectrometer via a Pico-Tip Emitter 360- $\mu$ m outer diameter $\times 20-\mu m$ inner diameter, 
10- $\mu \mathrm{m}$ tip (New Objective) heated at $300{ }^{\circ} \mathrm{C}$, and a spray voltage of $2.2 \mathrm{kV}$ was applied. The capillary temperature was set at $300^{\circ} \mathrm{C}$. The radio frequency ion funnel was set to $30 \%$. For DIA data acquisition, full scan mass spectrometry (MS) spectra with mass range 350-1650 $\mathrm{m} / \mathrm{z}$ were acquired in profile mode in the Orbitrap with resolution of $120,000 \mathrm{FWHM}$. The default charge state was set to 3+. The filling time was set at a maximum of $60 \mathrm{~ms}$ with a limitation of $3 \times 10^{6}$ ions. DIA scans were acquired with 40 mass window segments of differing widths across the MS1 mass range. Higher collisional dissociation fragmentation (stepped normalized collision energy; $25,27.5$, and $30 \%$ ) was applied and MS/MS spectra were acquired with a resolution of 30,000 FWHM with a fixed first mass of $200 \mathrm{~m} / \mathrm{z}$ after accumulation of $3 \times 10^{6}$ ions or after filling time of $35 \mathrm{~ms}$ (whichever occurred first). Datas were acquired in profile mode. For data acquisition and processing of the raw data Xcalibur 4.3 (Thermo) and Tune version 2.0 were used.

Mass Spectrometry data processing

\section{Data processing for dimethyl-labelled samples (Zebrafish and Rat neuronal} 912 differentiation) Software MaxQuant (version 1.5.3.28) was used to search the MS .raw data. For $D$. rerio the raw data were searched against the $D$. rerio UniProt database release: 2018_03, while for $R$. norvegicus the raw files from (Frese et al. 2017), were downloaded from PRIDE repository PXD005031 and searched against the UniProt $R$. norvegicus database release 2019_08. Both datasets were searched appending a list of common contaminants. The data were searched with the following modifications: Carbamidomethyl (C) (fixed) and Oxidation (M) and Acetyl (Protein N-term; variable). For D. rerio 2 labels, Light $L$ (DmethLys0 and DmethNterm0) and Heavy $\mathrm{H}$ (DmethLys4 and DmethNterm4) were selected representing the stem cell and neurons respectively. For the re-analysis of $R$. norvegicus data from Frese et al., 3 different labels were used: Light $L$ (DmethLys0 and DmethNterm0), Medium M, (DmethLys4 and DmethNterm4) and Heavy $\mathrm{H}$ (DmethLys8 and DmethNterm8). For identification, match between runs was selected with a match time window of 2 minutes, and an alignment time window of 20 minutes. The mass error tolerance for the full scan MS spectra was set at 20 ppm and for the MS/MS spectra at 0.5 Da. A maximum of two missed cleavages was allowed. Identifications were filtered at $1 \%$ FDR at both peptide and protein levels using a target-decoy strategy (Elias and Gygi 2007). From each experiment, iBAQ values (Schwanhäusser et al. 2011) and ratios between labels were extracted from the ProteinGroups.txt table. Differential expression analysis was performed using the mean of the normalized ratios between labels. The R package fdrtool (Strimmer 2008) was used to calculate $p$ values and $q$ values for the different comparisons, on the Log2 transformed mean ratios.

\section{Data processing for TMT10-plex data (mouse in vitro differentiation)} Waltham, MA, USA). raw files were searched against the fasta database (Uniprot Mus musculus database, reviewed entry only, release 2016_11) using Mascot v2.5.1 (Matrix Science) with the following settings: Enzyme was set to trypsin, with up to 1 missed cleavage. MS1 mass tolerance was set to $10 \mathrm{ppm}$ and MS2 to $0.5 \mathrm{Da}$. Carbamidomethyl cysteine was set as a fixed modification while oxidation of methionine and acetylation ( $\mathrm{N}$-term) were set as variable. Other modifications included the TMT-10plex modification from the quantification 
943 method used. The quantification method was set for reporter ions quantification with HCD and 944 MS3 (mass tolerance, 20ppm). False discovery rate for peptide-spectrum matches (PSMs) 945 was set to 0.01 using Percolator 13 (Brosch et al. 2009). Reporter ion intensity values for the 946 PSMs were exported and processed with procedures written in R (v.4.0.5) and R studio server 947 (v.1.2.5042 and 1.4.1106), as described in (Heinze et al. 2018). Briefly, PSMs mapping to 948 reverse or contaminant hits, or having a Mascot score below 15, or having reporter ion 949 intensities below $1 \mathrm{e} 3$ in all the relevant TMT channels were discarded. TMT channels 950 intensities from the retained PSMs were then log2 transformed, normalized and summarized into protein group quantities by taking the median value using MSnbase (Gatto and Lilley 2012). At least two unique peptides per protein were required for the identification and only those peptides with no missing values across all 10 channels were considered for quantification. Protein differential expression was evaluated using the limma package (Ritchie et al., 2015). Differences in protein abundances were statistically determined using the Student's $t$ test moderated by the empirical Bayes method. $P$ values were adjusted for multiple testing using the Benjamini-Hochberg method (FDR, denoted as "adj. p") (Benjamini and Hochberg, 1995).

\section{Data processing for DIA samples (SEC23A/B knockdowns)}

962

DIA libraries were created by searching the DIA runs using Spectronaut Pulsar (v13),

963 Biognosys, Zurich, Switzerland). The data were searched against species specific protein databases (Uniprot Mus musculus release 2016_01) with a list of common contaminants appended. The data were searched with the following modifications: carbamidomethyl $(\mathrm{C})$ as fixed modification, and oxidation (M), acetyl (protein $\mathrm{N}$-term). A maximum of 2 missed cleavages was allowed. The library search was set to $1 \%$ false discovery rate (FDR) at both protein and peptide levels. Libraries contained a total of 101,659 precursors, corresponding to 5708 and 6003 protein groups respectively. DIA data were then uploaded and searched against this spectral library using Spectronaut Professional (v.14.10) and default settings. Relative quantification was performed in Spectronaut for each pairwise comparison using the replicate samples from each condition using default settings, except: Data Filtering set to Qvalue sparse, and imputation to RunWise. Differential abundance testing was performed using a paired t-test between replicates. The data (candidate tables) and protein quantity data reports were then exported for further data analyses.

\section{Data processing for human neuronal differentiation data}

979

Protein identifications and LFQ intensity values (Log2) in cultured iPSCs, NPCs and differentiated neurons, were obtained from the original Supplementary table S2 published in (Djuric et al. 2017). Differential expression analysis between the different conditions was

\section{Data analysis}

\section{Analysis of paralog pairs during development and across tissues} pairs Pearson correlation coefficients. For the Human Tissue Atlas (Wang et al., 2019), transcript pairs. In all dataset only genes and proteins identified in at least 5 time-points / 
tissues were considered for correlation analysis. Coefficient of variations ( $\sigma$ / mean protein or transcript expression along time points / tissues) were also calculated for every gene in each datasets. Genes that have at least one paralog in the genome according to Ensembl Compara were labelled as 'Have Paralogs', and used for further analysis. From all the possible paralog pairs, 3 categories were created. The first one indicates all the possible paralog gene pairs, the second one indicates paralog pairs residing in the same protein complexes according to definitions from Ori et al., 2016, and the third one given by the exclusion between the two, indicating all other paralog pairs, namely paralog pairs that do not reside in the same complexes. For every paralog pair, the mean sequence identity was then calculated as the mean reciprocal identity retrieved from the Ensembl database. The relationship between sequence identity and co-expression between paralog pairs, was evaluated using Pearson $R$ correlation coefficient, and visualized through a Generalized Additive Model.

\section{Protein complex analysis during zebrafish development and across human tissues}

For each datasets, proteins were annotated with the different protein complex definitions. Only protein complexes with at least 5 subunits present in each of the dataset were retained for analysis. For each of these complexes, all the possible pairwise correlations between subunits were considered, and from those the median value was used to calculate a median complex co-expression. We defined stable and variable complexes using the top and bottom $25 \%$ of the distribution respectively. (1- median Perason correlation) was also used to define then a measure of protein complex stoichiometric variability, as shown in Fig1F/G. The distribution of correlations was then compared with a distribution of randomly assembled complexes of same size and complex members obtained by randomly assigning proteins/transcripts to complexes. For each dataset, the fraction of paralog pairs present was considered as the number of subunits that have at least one paralog in the genome divided by the total size of each protein complex. Finally for each subunit, we calculated expression correlation values with the other members of the same complex, taking the median of this value as a measure of co-expression for that specific subunit. Top and bottom $25 \%$ of the obtained distribution were used to define stoichiometrically stable or variable subunits, respectively.

\section{Paralog regulation during neuronal differentiation}

For each datasets, differentially expressed proteins between different conditions (Log 2 FoldChange $>0.58$ and adjusted $p$ value, or fdr tools $p$ value $<0.05$ ) were selected. Proteins were annotated as "Have Paralogs" if they had at least one paralog annotated in the genome. For each comparison, we then considered all possible paralog pairs present in the data and identified unique paralog pairs that displayed concerted regulation (same Log2 Fold Change sign for both paralogs) or opposite regulation (different Log2 Fold Change sign).

\section{Subunits co-expression analysis for neuronal differentiation data}

For calculating subunits stoichiometric variability we adapted a previously established pipeline (Gehring J, 2021). For each condition and datasets, only protein complexes that had at least 5 quantified subunits were considered. Then for each subunit in each complex, the median euclidean distance of fold change between that subunit and all other complex members was calculated. The distance obtained was compared with a distribution of distances for 2500 subunits from random complexes of equal size, obtained by randomly assigning proteins identified in the data to protein complexes. By comparing the two distributions we obtained a probability value for each subunit of observing lower distances with the complexes. Low p. values indicate high coexpression, denoted as stoichiometric stability, and vice versa. 
1038

1039

1040

1041

1042

1043

1044

1045

1046

1047

1048

1049

1050

1051

1052

1053

1054

1055

1056

1057

1058

1059

1060

1061

1062

1063

1064

1065

1066

1067

1068

1069

1070

1071

1072

1073

1074

1075

1076

1077

1078

1079

1080

\section{eggNOG mapping}

Fasta proteomes sequences used for MS protein quantification of the different dataset were annotated using emapper-2.1.4-2 (Cantalapiedra et al., 2021), based on eggNOG orthology data (Huerta-Cepas et al. 2019). Sequence searches were performed using the software MMseqs2 (Steinegger and Söding 2017). For each proteome eggNOG annotation was performed using default parameters.

\section{Conserved exchange of paralog proteins}

For each dataset, protein quantification values were used to calculate paralog ratios across conditions. The log2 paralog ratio between all possible quantified paralog pairs in each replicate was calculated for all the conditions tested. For each dataset, the significance of paralog ratio changes was assessed using the $R$ package limma (Ritchie et al. 2015) considering replicates information. We considered only ratio changes relative to the first time point of each neuronal differentiation dataset. For comparison across species each paralog pair was mapped to its relative eggNOG. Only paralog pairs where both entries could be mapped to a valid eggNOG were retained. After eggNOG mapping, shared eggNOG pairs between species were used to assess if specific paralog substitution were shared across different organisms, and for each specific comparison we combined the $p$ values using Fisher's combined probability test from the metaRNASeq R package (https://cran.rproject.org/web/packages/metaRNASeq/index.html). Combined $\mathrm{p}$ values were corrected for multiple testing using the Benjamin-Hochberg correction (Benjamini and Hochberg 1995). Since in some cases multiple proteins can map to the same eggNOG, for each pair and condition the mean value was considered for both ratio differences and $p$ values. From this analysis, we considered as "conserved" only paralog gene pairs identified in all species and whose $\log 2$ ratio changes were consistent in sign in at least 5 of the 7 neuronal differentiation comparisons, with combined adjusted $p<=0.05$.

\section{GO enrichment analysis}

Over representation analysis of $G O$ terms was performed with the $R$ package topGO (https://bioconductor.org/packages/release/bioc/html/topGO.html). Fisher test was used in order to estimate the expected proportion for different terms and obtain a $p$ value indicating the enrichment score for each specific GO term. Gene set enrichment analysis (GSEA) was performed with the topGO R package using a Kolmogorov-Smirnov test on the cumulative ranked distributions. For both enrichments $p$ values were adjusted using Hommel's correction, GOTerms were considered significant if their adjusted $p$ values were below the value of 0.05 . The R package rrvgo (https://ssayols.github.io/rrvgo/) was used in order to summarize and reduce redundancy of the enriched $\mathrm{GO}$ terms using default settings.

\section{Figure generation}

Data visualization was performed with $R$ (v.4.0.5) and $R$ studio server (Version 1.4.1106) using the ggplot2 package (Wickham 2009). Figure panels 1A, 2A, 4A were created with BioRender.com. 


\section{Bibliography}

1085

1086

1087

1088

1089

1090

1091

1092

1093

1094

1095

1096

1097

1098

1099

1100

1101

1102

1103

1104

1105

1106

1107

1108

1109

1110

1111

1112

1113

1114

1115

1116

1117

1118

1119

1120

1121

1122

1123

1124

1125

1126

1127

1128

1129

1130

1131

1132

1133

1134

1135
Arendt, Detlev, Jacob M. Musser, Clare V. H. Baker, Aviv Bergman, Connie Cepko, Douglas H. Erwin, Mihaela Pavlicev, et al. 2016. "The Origin and Evolution of Cell Types." Nature Reviews. Genetics 17 (12): 744-57.

Assis, Raquel, and Doris Bachtrog. 2015. "Rapid Divergence and Diversification of Mammalian Duplicate Gene Functions." BMC Evolutionary Biology 15 (July): 138.

Benjamini, Yoav, and Yosef Hochberg. 1995. "Controlling the False Discovery Rate: A Practical and Powerful Approach to Multiple Testing." Journal of the Royal Statistical Society: Series B (Methodological). https://doi.org/10.1111/j.2517-6161.1995.tb02031.x.

Boersema, Paul J., Reinout Raijmakers, Simone Lemeer, Shabaz Mohammed, and Albert J. R. Heck. 2009. "Multiplex Peptide Stable Isotope Dimethyl Labeling for Quantitative Proteomics." Nature Protocols 4 (4): 484-94.

Brohard-Julien, Solène, Vincent Frouin, Vincent Meyer, Smahane Chalabi, Jean-François Deleuze, Edith Le Floch, and Christophe Battail. 2021. "Region-Specific Expression of Young Small-Scale Duplications in the Human Central Nervous System." BMC Ecology and Evolution 21 (1): 59.

Brosch, Markus, Lu Yu, Tim Hubbard, and Jyoti Choudhary. 2009. "Accurate and Sensitive Peptide Identification with Mascot Percolator." Journal of Proteome Research 8 (6): 3176-81.

Brunet, Thibaut, and Nicole King. 2017. "The Origin of Animal Multicellularity and Cell Differentiation." Developmental Cell 43 (2): 124-40.

Buczak, Katarzyna, Joanna M. Kirkpatrick, Felicia Truckenmueller, Deolinda Santinha, Lino Ferreira, Stephanie Roessler, Stephan Singer, Martin Beck, and Alessandro Ori. 2020. "Spatially Resolved Analysis of FFPE Tissue Proteomes by Quantitative Mass Spectrometry." Nature Protocols 15 (9): 2956-79.

Cantalapiedra, Carlos P., Ana Hernández-Plaza, Ivica Letunic, Peer Bork, and Jaime Huerta-Cepas. n.d. "eggNOG-Mapper v2: Functional Annotation, Orthology Assignments, and Domain Prediction at the Metagenomic Scale." https://doi.org/10.1101/2021.06.03.446934.

Coppola, T., S. Magnin-Luthi, V. Perret-Menoud, S. Gattesco, G. Schiavo, and R. Regazzi. 2001. "Direct Interaction of the Rab3 Effector RIM with Ca2+ Channels, SNAP-25, and Synaptotagmin." The Journal of Biological Chemistry 276 (35): 32756-62.

Dandage, Rohan, and Christian R. Landry. 2019. "Paralog Dependency Indirectly Affects the Robustness of Human Cells." Molecular Systems Biology 15 (9): e8871.

De Kegel, Barbara, and Colm J. Ryan. 2019. "Paralog Buffering Contributes to the Variable Essentiality of Genes in Cancer Cell Lines." PLoS Genetics 15 (10): e1008466.

Djuric, Ugljesa, Deivid C. Rodrigues, Ihor Batruch, James Ellis, Patrick Shannon, and Phedias Diamandis. 2017. "Spatiotemporal Proteomic Profiling of Human Cerebral Development." Molecular \& Cellular Proteomics: MCP 16 (9): 1548-62.

Dominguez, Martin H., Albert E. Ayoub, and Pasko Rakic. 2013. "POU-III Transcription Factors (Brn1, Brn2, and Oct6) Influence Neurogenesis, Molecular Identity, and Migratory Destination of Upper-Layer Cells of the Cerebral Cortex." Cerebral Cortex 23 (11): 2632-43.

Durinck, Steffen, Paul T. Spellman, Ewan Birney, and Wolfgang Huber. 2009. "Mapping Identifiers for the Integration of Genomic Datasets with the R/Bioconductor Package biomaRt." Nature Protocols. https://doi.org/10.1038/nprot.2009.97.

Elias, Joshua E., and Steven P. Gygi. 2007. "Target-Decoy Search Strategy for Increased Confidence in Large-Scale Protein Identifications by Mass Spectrometry." Nature Methods. https://doi.org/10.1038/nmeth1019.

Ferrier, David E. K., and Peter W. H. Holland. 2001. "Ancient Origin of the Hox Gene Cluster." Nature Reviews Genetics. https://doi.org/10.1038/35047605.

Frese, Christian K., Marina Mikhaylova, Riccardo Stucchi, Violette Gautier, Qingyang Liu, 
Shabaz Mohammed, Albert J. R. Heck, A. F. Maarten Altelaar, and Casper C. Hoogenraad. 2017. "Quantitative Map of Proteome Dynamics during Neuronal Differentiation." Cell Reports 18 (6): 1527-42.

Gatto, Laurent, and Kathryn S. Lilley. 2012. "MSnbase-an R/Bioconductor Package for Isobaric Tagged Mass Spectrometry Data Visualization, Processing and Quantitation." Bioinformatics 28 (2): 288-89.

Genuth, Naomi R., and Maria Barna. 2018. "The Discovery of Ribosome Heterogeneity and Its Implications for Gene Regulation and Organismal Life." Molecular Cell 71 (3): 36474.

Gehring J. 2021. proteinProfiles: Protein Profiling. R package version 1.32 .0

Gerst, Jeffrey E. 2018. "Pimp My Ribosome: Ribosomal Protein Paralogs Specify Translational Control." Trends in Genetics. https://doi.org/10.1016/j.tig.2018.08.004. Guschanski, Katerina, Maria Warnefors, and Henrik Kaessmann. 2017. "The Evolution of Duplicate Gene Expression in Mammalian Organs." Genome Research 27 (9): 146174.

Hansson, Jenny, Mahmoud Reza Rafiee, Sonja Reiland, Jose M. Polo, Julian Gehring, Satoshi Okawa, Wolfgang Huber, Konrad Hochedlinger, and Jeroen Krijgsveld. 2012. "Highly Coordinated Proteome Dynamics during Reprogramming of Somatic Cells to Pluripotency." Cell Reports. https://doi.org/10.1016/j.celrep.2012.10.014.

Heinze, Ivonne, Martin Bens, Enrico Calzia, Susanne Holtze, Oleksandr Dakhovnik, Arne Sahm, Joanna M. Kirkpatrick, et al. 2018. "Species Comparison of Liver Proteomes Reveals Links to Naked Mole-Rat Longevity and Human Aging." BMC Biology 16 (1): 82.

Ho, Lena, Jehnna L. Ronan, Jiang Wu, Brett T. Staahl, Lei Chen, Ann Kuo, Julie Lessard, Alexey I. Nesvizhskii, Jeff Ranish, and Gerald R. Crabtree. 2009. "An Embryonic Stem Cell Chromatin Remodeling Complex, esBAF, Is Essential for Embryonic Stem Cell Self-Renewal and Pluripotency." Proceedings of the National Academy of Sciences of the United States of America 106 (13): 5181-86.

Huerta-Cepas, Jaime, Damian Szklarczyk, Davide Heller, Ana Hernández-Plaza, Sofia K. Forslund, Helen Cook, Daniel R. Mende, et al. 2019. "eggNOG 5.0: A Hierarchical, Functionally and Phylogenetically Annotated Orthology Resource Based on 5090 Organisms and 2502 Viruses." Nucleic Acids Research. https://doi.org/10.1093/nar/gky1085.

Ibn-Salem, Jonas, Enrique M. Muro, and Miguel A. Andrade-Navarro. 2017. "Co-Regulation of Paralog Genes in the Three-Dimensional Chromatin Architecture." Nucleic Acids Research 45 (1): 81-91.

Jain Goyal, Manu, Xiyan Zhao, Mariya Bozhinova, Karla Andrade-López, Cecilia de Heus, Sandra Schulze-Dramac, Michaela Müller-McNicoll, Judith Klumperman, and Julien Béthune. 2020. "A Paralog-Specific Role of COPI Vesicles in the Neuronal Differentiation of Mouse Pluripotent Cells." Life Science Alliance 3 (9). https://doi.org/10.26508/lsa.202000714.

Kaeser, Matthias D., Aaron Aslanian, Meng-Qiu Dong, John R. Yates 3rd, and Beverly M. Emerson. 2008. "BRD7, a Novel PBAF-Specific SWI/SNF Subunit, Is Required for Target Gene Activation and Repression in Embryonic Stem Cells." The Journal of Biological Chemistry 283 (47): 32254-63.

Kaessmann, Henrik. 2010. "Origins, Evolution, and Phenotypic Impact of New Genes." Genome Research 20 (10): 1313-26.

Kang, Min Ah, Jong-Tae Kim, Joo Heon Kim, Soo-Young Kim, Young Ho Kim, Young II Yeom, Younghee Lee, and Hee Gu Lee. 2009. "Upregulation of the Cycline Kinase Subunit CKS2 Increases Cell Proliferation Rate in Gastric Cancer." Journal of Cancer Research and Clinical Oncology 135 (6): 761-69.

Khoriaty, Rami, Geoffrey G. Hesketh, Amélie Bernard, Angela C. Weyand, Dattatreya Mellacheruvu, Guojing Zhu, Mark J. Hoenerhoff, et al. 2018. "Functions of the COPII Gene Paralogs SEC23A and SEC23B Are Interchangeable in Vivo." Proceedings of the 
National Academy of Sciences of the United States of America 115 (33): E7748-57.

Lauria, Fabio, Paola Bernabò, Toma Tebaldi, Ewout Joan Nicolaas Groen, Elena Perenthaler, Federica Maniscalco, Annalisa Rossi, et al. 2020. "SMN-Primed Ribosomes Modulate the Translation of Transcripts Related to Spinal Muscular Atrophy." Nature Cell Biology 22 (10): 1239-51.

Lin, Lingqing, Zanxi Fang, Huayue Lin, Hanyu You, Jiajia Wang, Yuanhui Su, Fen Wang, and Zhong-Ying Zhang. 2016. "Depletion of Cks1 and Cks2 Expression Compromises Cell Proliferation and Enhance Chemotherapy-Induced Apoptosis in HepG2 Cells." Oncology Reports 35 (1): 26-32.

Liu, Hong, Ariane Beauvais, Adam N. Baker, Catherine Tsilfidis, and Rashmi Kothary. 2011. "Smn Deficiency Causes Neuritogenesis and Neurogenesis Defects in the Retinal Neurons of a Mouse Model of Spinal Muscular Atrophy." Developmental Neurobiology. https://doi.org/10.1002/dneu.20840.

Lynch, Michael, and John S. Conery. 2003. "The Origins of Genome Complexity." Science. https://doi.org/10.1126/science.1089370.

Makova, K. D. 2003. "Divergence in the Spatial Pattern of Gene Expression Between Human Duplicate Genes." Genome Research. https://doi.org/10.1101/gr.1133803.

Mertens, Jerome, Dylan Reid, Shong Lau, Yongsung Kim, and Fred H. Gage. 2018. "Aging in a Dish: iPSC-Derived and Directly Induced Neurons for Studying Brain Aging and Age-Related Neurodegenerative Diseases." Annual Review of Genetics. https://doi.org/10.1146/annurev-genet-120417-031534.

Nithianantharajah, J., N. H. Komiyama, A. McKechanie, M. Johnstone, D. H. Blackwood, D. St Clair, R. D. Emes, et al. 2013. "Synaptic Scaffold Evolution Generated Components of Vertebrate Cognitive Complexity." Nature Neuroscience 16 (1). https://doi.org/10.1038/nn.3276.

Ohno, Susumu. 2013. Evolution by Gene Duplication. Springer Science \& Business Media.

Ori, Alessandro, Murat Iskar, Katarzyna Buczak, Panagiotis Kastritis, Luca Parca, Amparo Andrés-Pons, Stephan Singer, Peer Bork, and Martin Beck. 2016. "Spatiotemporal Variation of Mammalian Protein Complex Stoichiometries." Genome Biology 17 (March): 47.

Padawer, T., R. E. Leighty, and D. Wang. 2012. "Duplicate Gene Enrichment and Expression Pattern Diversification in Multicellularity." Nucleic Acids Research. https://doi.org/10.1093/nar/gks464.

Pelicci, Giuliana, Flavia Troglio, Alessandra Bodini, Rosa Marina Melillo, Valentina Pettirossi, Laura Coda, Antonio De Giuseppe, Massimo Santoro, and Pier Giuseppe Pelicci. 2002. "The Neuron-Specific Rai (ShcC) Adaptor Protein Inhibits Apoptosis by Coupling Ret to the Phosphatidylinositol 3-kinase/Akt Signaling Pathway." Molecular and Cellular Biology 22 (20): 7351-63.

Perez-Riverol, Yasset, Attila Csordas, Jingwen Bai, Manuel Bernal-Llinares, Suresh Hewapathirana, Deepti J. Kundu, Avinash Inuganti, et al. 2019. "The PRIDE Database and Related Tools and Resources in 2019: Improving Support for Quantification Data." Nucleic Acids Research 47 (D1): D442-50.

Peri, Francesca, and Christiane Nüsslein-Volhard. 2008. "Live Imaging of Neuronal Degradation by Microglia Reveals a Role for v0-ATPase a1 in Phagosomal Fusion in Vivo." Cell 133 (5): 916-27.

Ritchie, Matthew E., Belinda Phipson, Di Wu, Yifang Hu, Charity W. Law, Wei Shi, and Gordon K. Smyth. 2015. "Limma Powers Differential Expression Analyses for RNASequencing and Microarray Studies." Nucleic Acids Research 43 (7): e47.

Romanov, Natalie, Michael Kuhn, Ruedi Aebersold, Alessandro Ori, Martin Beck, and Peer Bork. 2019. "Disentangling Genetic and Environmental Effects on the Proteotypes of Individuals." Cell 177 (5): 1308-18.e10.

Scharaw, Sandra, Murat Iskar, Alessandro Ori, Gaelle Boncompain, Vibor Laketa, Ina Poser, Emma Lundberg, et al. 2016. "The Endosomal Transcriptional Regulator RNF11 Integrates Degradation and Transport of EGFR." The Journal of Cell Biology 215 (4): 543-58. 
Schmidt, Ewoud R. E., Justine V. Kupferman, Michelle Stackmann, and Franck Polleux. 2019. "The Human-Specific Paralogs SRGAP2B and SRGAP2C Differentially Modulate SRGAP2A-Dependent Synaptic Development." Scientific Reports 9 (1): 18692.

Schwanhäusser, Björn, Dorothea Busse, Na Li, Gunnar Dittmar, Johannes Schuchhardt, Jana Wolf, Wei Chen, and Matthias Selbach. 2011. "Global Quantification of Mammalian Gene Expression Control." Nature. https://doi.org/10.1038/nature10098.

Shi, Zhen, Kotaro Fujii, Kyle M. Kovary, Naomi R. Genuth, Hannes L. Röst, Mary N. Teruel, and Maria Barna. 2017. "Heterogeneous Ribosomes Preferentially Translate Distinct Subpools of mRNAs Genome-Wide." Molecular Cell 67 (1): 71-83.e7.

Slavov, Nikolai, Stefan Semrau, Edoardo Airoldi, Bogdan Budnik, and Alexander van Oudenaarden. 2015. "Differential Stoichiometry among Core Ribosomal Proteins." Cell Reports 13 (5): 865-73.

Son, Esther Y., and Gerald R. Crabtree. 2014. "The Role of BAF (mSWI/SNF) Complexes in Mammalian Neural Development." American Journal of Medical Genetics. Part C, Seminars in Medical Genetics 166C (3): 333-49.

Soria, Patricia S., Kriston L. McGary, and Antonis Rokas. 2014. "Functional Divergence for Every Paralog." Molecular Biology and Evolution 31 (4): 984-92.

Steinegger, Martin, and Johannes Söding. 2017. "MMseqs2 Enables Sensitive Protein Sequence Searching for the Analysis of Massive Data Sets." Nature Biotechnology. https://doi.org/10.1038/nbt.3988.

Strimmer, Korbinian. 2008. "Fdrtool: A Versatile R Package for Estimating Local and Tail Area-Based False Discovery Rates." Bioinformatics 24 (12): 1461-62.

Sugitani, Y. 2002. "Brn-1 and Brn-2 Share Crucial Roles in the Production and Positioning of Mouse Neocortical Neurons." Genes \& Development. https://doi.org/10.1101/gad.978002.

Suzuki, Ikuo K., David Gacquer, Roxane Van Heurck, Devesh Kumar, Marta Wojno, Angéline Bilheu, Adèle Herpoel, et al. 2018. "Human-Specific NOTCH2NL Genes Expand Cortical Neurogenesis through Delta/Notch Regulation." Cell 173 (6): 1370_ 84.e16.

Thompson, Nicola A., Marco Ranzani, Louise van der Weyden, Vivek lyer, Victoria Offord, Alastair Droop, Fiona Behan, et al. 2021. "Combinatorial CRISPR Screen Identifies Fitness Effects of Gene Paralogues." Nature Communications 12 (1): 1302.

Toufighi, Kiana, Jae-Seong Yang, Nuno Miguel Luis, Salvador Aznar Benitah, Ben Lehner, Luis Serrano, and Christina Kiel. 2015. "Dissecting the Calcium-Induced Differentiation of Human Primary Keratinocytes Stem Cells by Integrative and Structural Network Analyses." PLoS Computational Biology 11 (5): e1004256.

Wang, Dongxue, Basak Eraslan, Thomas Wieland, Björn Hallström, Thomas Hopf, Daniel Paul Zolg, Jana Zecha, et al. 2019. "A Deep Proteome and Transcriptome Abundance Atlas of 29 Healthy Human Tissues." Molecular Systems Biology 15 (2): e8503.

White, Richard J., John E. Collins, Ian M. Sealy, Neha Wali, Christopher M. Dooley, Zsofia Digby, Derek L. Stemple, et al. 2017. "A High-Resolution mRNA Expression Time Course of Embryonic Development in Zebrafish." eLife 6 (November). https://doi.org/10.7554/eLife.30860.

Wickham, Hadley. 2009. ggplot2: Elegant Graphics for Data Analysis. Springer Science \& Business Media.

Xue, Yutong, Julie C. Canman, Cheol Soon Lee, Zuqin Nie, Dafeng Yang, G. Tony Moreno, Mary K. Young, E. D. Salmon, and Weidong Wang. 2000. "The Human SWI/SNF-B Chromatin-Remodeling Complex Is Related to Yeast Rsc and Localizes at Kinetochores of Mitotic Chromosomes." Proceedings of the National Academy of Sciences of the United States of America 97 (24): 13015.

Yates, Andrew D., Premanand Achuthan, Wasiu Akanni, James Allen, Jamie Allen, Jorge Alvarez-Jarreta, M. Ridwan Amode, et al. 2020. "Ensembl 2020." Nucleic Acids Research 48 (D1): D682-88.

Zeng, Yonglun, Kin Pan Chung, Baiying Li, Ching Man Lai, Sheung Kwan Lam, Xiangfeng Wang, Yong Cui, et al. 2015. "Unique COPII Component AtSar1a/AtSEC23A Pair Is 
bioRxiv preprint doi: https://doi.org/10.1101/2021.07 22.453347; this version posted July 23, 2021. The copyright holder for this preprint (which was not certified by peer review) is the author/funder, who has granted bioRxiv a license to display the preprint in perpetuity. It is made available under aCC-BY-NC-ND 4.0 International license.

Required for the Distinct Function of Protein ER Export in Arabidopsis Thaliana." Proceedings of the National Academy of Sciences of the United States of America 112 (46): 14360-65.

Zhu, Min, Jiayi Tao, Matthew P. Vasievich, Wei Wei, Guojing Zhu, Rami N. Khoriaty, and Bin Zhang. 2015. "Neural Tube Opening and Abnormal Extraembryonic Membrane Development in SEC23A Deficient Mice." Scientific Reports 5 (October): 15471. 